\title{
THERMO-ELASTIC TRIANGULAR SANDWICH ELEMENT FOR THE COMPLETE STRESS FIELD BASED ON A SINGLE-LAYER THEORY
}

\author{
M. Das, ${ }^{*}$ A. Barut, ${ }^{\dagger}$ E. Madenci ${ }^{\ddagger}$ \\ The University of Arizona, Tucson, AZ 85721 \\ D. R. Ambur ${ }^{\S}$ \\ NASA Langley Research Center, Hampton, VA 23665
}

\begin{abstract}
This study presents a new triangular finite element for modeling thick sandwich panels, subjected to thermo-mechanical loading, based on a $\{3,2\}$-order singlelayer plate theory. A hybrid energy functional is employed in the derivation of the element because of a $C^{1}$ interelement continuity requirement. The single-layer theory is based on five weighted-average field variables arising from the cubic and quadratic representations of the in-plane and transverse displacement fields, respectively. The variations of temperature and distributed loading acting on the top and bottom surfaces are non-uniform. The temperature varies linearly through the thickness.
\end{abstract}

\section{Introduction}

The analysis of sandwich constructions with anisotropic composite facings for thermal stresses is of primary importance for the efficient design of high-speed aircraft and missiles. Subjected to hot environments, an analysis of such complex vehicles made of sandwich constructions can be performed by employing standard three-dimensional finite elements. However, this method is computationally demanding and very costly. An alternative to using standard elements is the employment of finite elements based on either a single-layer or discrete-layer plate theory in order to eliminate the discretization in the transverse direction. Concerning mechanical loading only, Das et al. ${ }^{1}$ provided an extensive review of the previous studies and introduced a new triangular finite element based on a single-layer theory.

A majority of the previous work concerning only thermal loading utilized the first-order shear deformation theory (FSDT). Extensive reviews of this theory, in

\footnotetext{
*Graduate Research Assistant, Department of Aerospace and Mechanical Engineering, Student Member AIAA.

${ }^{\dagger}$ Assistant Research Professor, Department of Aerospace and Mechanical Engineering.

†Professor, Department of Aerospace and Mechanical Engineering, Member AIAA.

${ }^{\S}$ Head, Mechanics and Durability Branch. Associate Fellow AIAA.

Copyright (C) 2004 by the American Institute of Aeronautics and Astronautics. All rights reserved.
}

the context of linear and non-linear finite element analysis, can be found in Refs. [2]-[5]. Although the FSDT provides acceptable mid-plane displacements, as well as in-plane stress components, it fails to capture the accurate behavior of transverse stresses, especially when analyzing sandwich constructions, which may have extremely different mechanical and thermal properties.

In order to improve the FSDT, Rolfes et al. ${ }^{6}$ developed a post-processing technique for computation of the transverse stresses by integrating the equilibrium equations in conjunction with a predictor-corrector approach when analyzing laminates. Park and $\mathrm{Kim}^{7}$ also introduced an approach to improve the FSDT by a two-phase analysis process. The first phase involves computation of the displacement components by integrating the transverse strains from the finite element analysis of FSDT. The second phase involves updating the force vector based on the non-linear part of the displacement components for further finite element analysis. Thankam et $\mathrm{al}^{8}{ }^{8}$ developed a material finite element for which the displacement approximation includes material properties, as well as the element coordinates. The expressions for the in-plane displacements are obtained from modified equilibrium equations.

Kant and Khare ${ }^{9}$ employed a $\{3,0\}$-order plate theory with cubic variation for in-plane displacements and a uniform transverse displacement. This theory does not require shear correction factors, and the resulting element utilizes a $C^{0}$ isoparametric interpolation function. However, as discussed by Naganarayan et al., ${ }^{10}$ this type of element is prone to error in the case of thermo-elastic loading. The error is traced to the nonparticipation and/or inconsistent participation of the higher-order terms of certain components of the strain fields in the total potential. In the case of thick laminates and sandwich constructions, the major shortcoming of this theory is the assumption of uniform transverse deformation. Employing the $\{1,2\}$ single-layer theory and exact solution, Tessler et al. ${ }^{11}$ showed that the transverse deformation is not uniform. Although the single-layer plate theory captures the non-uniform transverse deformation, it fails to capture the discontinuous slope of the in-plane and transverse displacement components in the thickness direction. 
The zig-zag theories and layer-wise theories eliminate this shortcoming of the single-layer theory. These theories enforce the continuity of in-plane displacements and transverse shear stresses at each layer interface, as well as the zero transverse shear stress at the top and bottom surfaces. Ali et al., ${ }^{12}$ Kapuria et al., ${ }^{13}$ and Xiaoping and Liangxin ${ }^{14}$ extended such theories to investigate the response of laminates and sandwich beams under thermal loading. Although layer-wise theories are suitable for the development of new finite elements, they become computationally expensive for sandwich structures containing multi-layered face sheets.

In order to take advantage of the computational efficiency of the single-layer theory, this study presents a new triangular sandwich finite element for thermoelastic analysis based on a hybrid-stress finite element formulation while employing the $\{3,2\}$-order singlelayer plate theory developed by Das et al. ${ }^{1}$ It captures both the transverse stresses and the discontinuous slope of the in-plane and transverse displacement components in the thickness direction by post-processing procedures. This element is validated by comparison against the exact solution for a simply supported laminate and a sandwich construction subjected to non-uniform temperature distribution.

\section{Element Description}

The sandwich element is composed of two face sheets with a soft core in between, as shown in Fig. 1. The mid-plane of the triangular sandwich element coincides with the $(x, y)$ plane of the element coordinate system. The origin of the element coordinate system is located at a node, and the $x$-axis is directed along an edge. The vector $\mathbf{n}_{k}$ represents the unit normal to the $k^{\text {th }}$ edge. The element has a uniform thickness of $2 h$. The thicknesses of the core and face sheets are denoted by $h_{c}$ and $h_{f}$, respectively. The face sheets, as well as the core, can be composed of homogeneous, elastic, orthotropic material layers. Each ply forming the face sheets has elastic moduli $E_{11}, E_{22}$, and $E_{33}$; shear moduli $G_{12}, \mathrm{G}_{13}$, and $G_{23}$; Poisson's ratios $v_{12}, v_{13}$, and $v_{23}$; and thermal expansion coefficients $\alpha_{1}, \alpha_{2}$ and $\alpha_{3}$, where subscripts 1 and 2 are the material coordinates. For an isotropic core material, the Young's modulus, Poisson's ratio, and thermal expansion coefficient are defined as $E_{c}, v_{c}$, and $\alpha_{c}$, respectively.

As shown in Fig. 1, the sandwich element is subjected to arbitrarily distributed loading acting on its upper and lower surfaces. The load distributions acting in the direction normal to the upper and lower surfaces are denoted by $q^{+}(x, y)$ and $q^{-}(x, y)$, respectively. The concentrated forces in an arbitrary direction are also considered as part of the external loading. The top

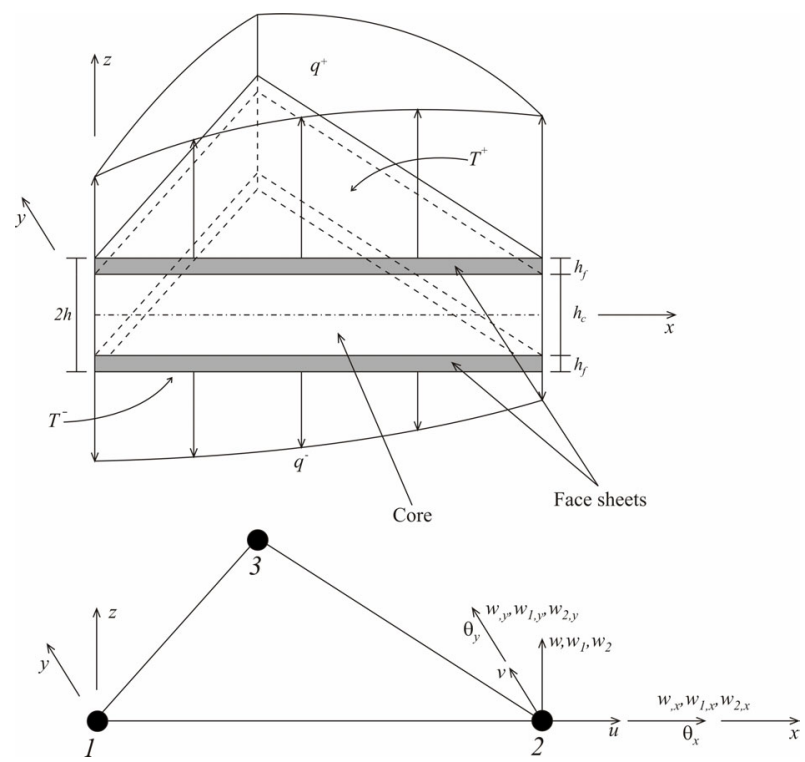

Fig. 1 Geometric description of the thick sandwich plate element.

and bottom surfaces of the element are exposed to the temperature variation of $T^{+}(x, y)$ and $T^{-}(x, y)$, respectively, The temperature varies linearly through the thickness. Furthermore, the sandwich element is subjected to arbitrary kinematic boundary conditions.

As shown in Fig. 1, this triangular element contains 13 degrees of freedom at each node. These degrees of freedom consist of two in-plane displacements $(u, v)$, two out-of-plane rotations $\left(\theta_{x}, \theta_{y}\right)$, and three transverse nodal displacements $\left(w, w_{1}, w_{2}\right)$ and their derivatives $\left(w_{, x}, w_{, y}, w_{1, x}, w_{1, y}, w_{2, x}, w_{2, y}\right)$. The weighted-average in-plane displacement components in the $x$ - and $y$ directions are denoted by $u$ and $v$, respectively. The weighted-average transverse displacement is denoted by $w$. The weighted-average bending rotations about the negative $x$ - and positive $y$-axes are denoted by $\theta_{x}$ and $\theta_{y}$, respectively. Their positive sign convention is shown in Fig. 1. The transverse displacements, $w_{1}$ and $w_{2}$ (not weighted-averaged), represent the symmetric and anti-symmetric expansion modes through the thickness of the element.

\section{Higher-Order $\{3,2\}$ Plate Theory}

The $\{3,2\}$-order plate theory implies that the in-plane displacement components vary cubically and the transverse displacement component varies quadratically across the thickness of the panel. At any point in the panel, the in-plane displacement components in the $x$ and $y$-directions are represented by $u_{x}(x, y, z)$ and $u_{y}(x, y, z)$, respectively, and the transverse displacement component by $u_{z}(x, y, z)$. In accordance with the 
$\{3,2\}$ plate theory, the displacement components of the sandwich panel are defined in the form

$$
\begin{aligned}
u_{x}(x, y, z) & =u_{0}(x, y)+u_{1}(x, y) \zeta \\
& +u_{2}(x, y) \zeta^{2}+u_{3}(x, y) \zeta^{3} \\
u_{y}(x, y, z) & =v_{0}(x, y)+v_{1}(x, y) \zeta \\
+ & v_{2}(x, y) \zeta^{2}+v_{3}(x, y) \zeta^{3} \\
u_{z}(x, y, z) & =w(x, y)+w_{1}(x, y) \zeta \\
& +w_{2}(x, y)\left(\zeta^{2}-1 / 5\right)
\end{aligned}
$$

where $\zeta=z / h$ is the normalized thickness and varies in the range $-1 \leq \zeta \leq 1$.

As introduced by Tessler, ${ }^{15}$ the weighted-average quantities through the thickness of the panel are defined as

$$
\begin{gathered}
(u(x, y), v(x, y))=\frac{1}{2 h} \int_{-h}^{h}\left(u_{x}(x, y, z), u_{y}(x, y, z)\right) d z \\
\left(\theta_{x}(x, y), \theta_{y}(x, y)\right) \\
=\frac{3}{2 h^{3}} \int_{-h}^{h}\left(u_{y}(x, y, z), u_{x}(x, y, z)\right) z d z \\
w(x, y)=\frac{3}{4 h} \int_{-h}^{h} u_{z}(x, y, z)\left(1-\zeta^{2}\right) d z
\end{gathered}
$$

Enforcing the definition for weighted-average kinematic variables results in the expressions for $u_{i}$ and $v_{i}$ $(i=2,3)$ in terms of the weighted-average kinematic variables, $u, v, \theta_{x}$, and $\theta_{y}$, and the unknown coefficients, $u_{i}$ and $v_{i}(i=0,1)$. The unknown coefficients, $u_{i}$ and $v_{i} \quad(i=0,1)$, are determined by imposing the condition of zero transverse shear strains on the top and bottom surfaces of the sandwich panel.

After determining the coefficients $u_{i}$ and $v_{i}(i=0,1$, $2,3)$, the displacement components, $u_{x}$ and $u_{y}$, are expressed in terms of the weighted-average quantities $\left(u, v, w, \theta_{x}, \theta_{y}\right)$ as

$$
\begin{aligned}
u_{x}(x, y, z) & =u(x, y)+h \zeta \theta_{y}(x, y) \\
& +\left(\frac{1}{6}-\frac{\zeta^{2}}{2}\right) h w_{1, x}(x, y) \\
& +h\left(\frac{\zeta}{5}-\frac{\zeta^{3}}{3}\right)\left[\frac{5}{4}\left(\theta_{y}(x, y)+w_{, x}(x, y)\right)\right. \\
& \left.+w_{2, x}(x, y)\right]
\end{aligned}
$$

$$
\begin{aligned}
u_{y}(x, y, z) & =v(x, y)+h \zeta \theta_{x}(x, y) \\
+ & \left(\frac{1}{6}-\frac{\zeta^{2}}{2}\right) h w_{1, y}(x, y) \\
+ & h\left(\frac{\zeta}{5}-\frac{\zeta^{3}}{3}\right)\left[\frac{5}{4}\left(\theta_{x}(x, y)+w_{, y}(x, y)\right)\right. \\
& \left.+w_{2, y}(x, y)\right]
\end{aligned}
$$

Substituting for the displacement components from Eqs. (3) and (1c) in the strain-displacement relations of the elasticity theory, the strain components can be expressed in the form

$$
\begin{aligned}
\varepsilon_{\alpha \beta} & ={ }^{(0)} \varepsilon_{\alpha \beta}+h \zeta^{(0)} \kappa_{\alpha \beta} \\
& +\left(\frac{1}{6}-\frac{\zeta^{2}}{2}\right){ }^{(1)} \varepsilon_{\alpha \beta} \quad(\alpha, \beta=x, y) \\
& +h\left(\frac{\zeta}{5}-\frac{\zeta^{3}}{3}\right){ }^{(1)} \kappa_{\alpha \beta} \\
\varepsilon_{z z} & ={ }^{(0)} \varepsilon_{z z}+2 h \zeta^{(0)} \kappa_{z z} \\
\left(\gamma_{y z}, \gamma_{x z}\right) & =\frac{5}{4}\left(1-\zeta^{2}\right)\left({ }^{(0)} \gamma_{y z},{ }^{(0)} \gamma_{x z}\right)
\end{aligned}
$$

in which the resultant strains and curvatures are defined as

$$
\begin{gathered}
{ }^{(0)} \varepsilon_{x x}=u_{, x}, \quad{ }^{(0)} \varepsilon_{y y}=v_{, y}, \\
{ }^{(0)} \varepsilon_{z z}=w_{1} / h, \quad{ }^{(0)} \gamma_{x y}=u_{, y}+v_{, x} \\
{ }^{(1)} \varepsilon_{x x}=h w_{, x x}, \quad{ }^{(1)} \varepsilon_{y y}=h w_{, y y}, \quad{ }^{(1)} \gamma_{x y}=2 h w_{, x y} \\
{ }^{(0)} \kappa_{x x}=\theta_{y, x}, \quad{ }^{(0)} \kappa_{y y}=\theta_{x, y} \\
{ }^{(0)} \kappa_{z z}=w_{2} / h^{2}, \quad{ }^{(0)} \kappa_{x y}=\theta_{x, x}+\theta_{y, y} \\
{ }^{(1)} \kappa_{x x}=\frac{5}{4}\left(\theta_{y, x}+w_{, x x}\right)+w_{2, x x} \\
{ }^{(1)} \kappa_{y y}=\frac{5}{4}\left(\theta_{x, y}+w_{, y y}\right)+w_{2, y y} \\
\kappa_{x y}=\frac{5}{4}\left(\theta_{x, x}+\theta_{y, y}+2 w_{, x y}\right)+2 w_{2, x y} \\
{ }^{(0)} \gamma_{y z}=w_{, y}+\theta_{x}, \quad{ }^{(0)} \gamma_{x z}=w_{, x}+\theta_{y}
\end{gathered}
$$

Unlike the conventional form of the relationship between the stress, $\sigma_{i j}$, and the strain, $\varepsilon_{i j}$, components, an alternative "mixed" form of the constitutive equations is employed as 


$$
\begin{aligned}
& \left\{\begin{array}{l}
\sigma_{x x}^{(k)} \\
\sigma_{y y}^{(k)} \\
\varepsilon_{z z}^{(k)} \\
\sigma_{y z}^{(k)} \\
\sigma_{x z}^{(k)} \\
\sigma_{x y}^{(k)}
\end{array}\right\}=\left[\begin{array}{llllll}
\bar{C}_{11} & \bar{C}_{12} & R_{13} & 0 & 0 & \bar{C}_{16} \\
\bar{C}_{12} & \bar{C}_{22} & R_{23} & 0 & 0 & \bar{C}_{26} \\
-R_{13} & -R_{23} & S_{33} & 0 & 0 & -R_{63} \\
0 & 0 & 0 & C_{44} & C_{45} & 0 \\
0 & 0 & 0 & C_{45} & C_{55} & 0 \\
\bar{C}_{16} & \bar{C}_{26} & R_{63} & 0 & 0 & \bar{C}_{66}
\end{array}\right]^{(k)} \\
& \times\left\{\begin{array}{l}
\varepsilon_{x x} \\
\varepsilon_{y y} \\
\sigma_{z z} \\
\gamma_{y z} \\
\gamma_{x z} \\
\gamma_{x y}
\end{array}\right\}-\Delta T\left[\begin{array}{ccccc}
\bar{C}_{11} & \bar{C}_{12} & \bar{C}_{16} & 0 \\
\bar{C}_{12} & \bar{C}_{22} & \bar{C}_{26} & 0 \\
-R_{13} & -R_{23} & -R_{63} & -1 \\
0 & 0 & 0 & 0 \\
0 & 0 & 0 & 0 \\
\bar{C}_{16} & \bar{C}_{26} & \bar{C}_{66} & 0
\end{array}\right]^{(k)}\left\{\begin{array}{l}
\alpha_{x x} \\
\alpha_{y y} \\
\alpha_{x y} \\
\alpha_{z z}
\end{array}\right\}
\end{aligned}
$$

in which the superscript $(k)$ indicates that the quantities are obtained from the stress-strain relations in the $k^{\text {th }}$ layer, and strain and stress quantities without the superscript on the right-hand side of Eq. (6) represent through-the-thickness behavior. The components of the material property matrix are defined as

$$
\begin{gathered}
\bar{C}_{i j}^{(k)}=C_{i j}^{(k)}-R_{i 3}^{(k)} C_{j 3}^{(k)} \quad(i, j=1,2,6) \\
R_{i 3}^{(k)}=C_{i 3}^{(k)} S_{33}^{(k)} \text { with } S_{33}^{(k)}=\frac{1}{C_{33}^{(k)}} \quad(i=1,2,6)
\end{gathered}
$$

The temperature change from a reference state, $\Delta T$, is assumed to vary linearly through the thickness and it has a non-uniform variation defined by $T(x, y)$ such that

$$
\Delta T=\left(\bar{T}_{1}+\bar{T}_{2} \zeta\right) T(x, y)
$$

where $\bar{T}_{1}$ and $\bar{T}_{2}$ can be expressed in terms of the surface temperatures at the top and bottom surfaces, i.e.,

$$
\begin{aligned}
& \bar{T}_{1}=\frac{1}{2}\left(\frac{T^{+}(x, y)+T^{-}(x, y)}{T(x, y)}\right) \\
& \bar{T}_{2}=\frac{1}{2}\left(\frac{T^{+}(x, y)-T^{-}(x, y)}{T(x, y)}\right)
\end{aligned}
$$

The transverse normal strain in the $k^{\text {th }}$ layer can then be expressed as

$$
\begin{aligned}
\varepsilon_{z z}^{(k)}= & -R_{13}^{(k)} \varepsilon_{x x}-R_{23}^{(k)} \varepsilon_{y y}-R_{63}^{(k)} \gamma_{x y}+S_{33}^{(k)} \sigma_{z z} \\
& +\Delta T\left(R_{13}^{(k)} \alpha_{x x}^{(k)}+R_{23}^{(k)} \alpha_{y y}^{(k)}+R_{63}^{(k)} \alpha_{x y}^{(k)}\right)
\end{aligned}
$$

in which the continuous through-the-thickness variations of $\varepsilon_{\alpha \alpha}$ and $\gamma_{\alpha \beta} \quad(\alpha, \beta=x, y)$ are known explicitly, but not that of $\sigma_{z z}$. In order to achieve a continu- ous through-the-thickness distribution of the transverse normal stress, a cubic polynomial is assumed for $\sigma_{z z}$ as

$$
\sigma_{z z}(x, y, z)=\sigma_{z z 0}(x, y)+\phi(\xi) \sigma_{z z 1}(x, y)
$$

where $\phi(\xi)=\zeta-\zeta^{3} / 3$ and $\sigma_{z z 0}$ and $\sigma_{z z 1}$ are unknown functions of $x$ and $y$. The unknown functions of the transverse stress component, $\sigma_{z z 0}$ and $\sigma_{z z 1}$, are determined such that the transverse strain component $\varepsilon_{z z}^{(k)}$ becomes the least-squares equivalent of the kinematic definition of the transverse normal strain component (i.e., $\varepsilon_{z z}=u_{z, z}$ ). This is achieved by minimizing the total error between $\varepsilon_{z z}^{(k)}$ and $\varepsilon_{z z}$ through the thickness of the panel, i.e.,

$$
\min \left[\int_{-h}^{h}\left(\varepsilon_{z z}^{(k)}-u_{z, z}\right)^{2} d z\right]
$$

Minimization of Eq. (12) with respect to $\sigma_{z z 0}$ and $\sigma_{\mathrm{zz} 1}$ results in the expression for $\sigma_{z z 0}$ and $\sigma_{z z 1}$ in the form

$$
\left\{\begin{array}{c}
\sigma_{z z 0} \\
\sigma_{z z 1}
\end{array}\right\}=\left[\begin{array}{ll}
\mathbf{q}_{\varepsilon}^{\prime} & \mathbf{q}_{\kappa}^{\prime} \\
\mathbf{q}_{\varepsilon}^{\prime \prime} & \mathbf{q}_{\kappa}^{\prime \prime}
\end{array}\right]\left\{\begin{array}{l}
\grave{\mathbf{o}} \\
\mathbf{\kappa}
\end{array}\right\}+\left\{\begin{array}{l}
r_{1} \\
r_{2}
\end{array}\right\} T(x, y)
$$

in which the known vectors $\mathbf{q}_{\alpha}^{\prime}$ and $\mathbf{q}_{\alpha}^{\prime \prime}(\alpha=\varepsilon, \kappa)$ are defined as

$$
\begin{aligned}
& \mathbf{q}_{a}^{\prime}=\left\{\begin{array}{llll}
q_{1 \alpha}^{\prime} & q_{2 \alpha}^{\prime} & \ldots & q_{7 \alpha}^{\prime}
\end{array}\right\} \\
& \mathbf{q}_{\alpha}^{\prime \prime}=\left\{\begin{array}{llll}
q_{1 \alpha}^{\prime \prime} & q_{2 \alpha}^{\prime \prime} & \ldots & q_{7 \alpha}^{\prime \prime}
\end{array}\right\} \quad(\alpha=\varepsilon, \kappa)
\end{aligned} \quad(\alpha)
$$

with their explicit expressions given in Appendix A. The resultant strain and curvature vectors are defined by

$$
\begin{aligned}
\mathbf{o} \bar{e} & =\left\{{ }^{(0)} \varepsilon_{x x},{ }^{(0)} \varepsilon_{y y},{ }^{(0)} \varepsilon_{z z},{ }^{(0)} \gamma_{x y},{ }^{(1)} \varepsilon_{x x},{ }^{(1)} \varepsilon_{y y},{ }^{(1)} \gamma_{x y}\right\} \\
\boldsymbol{\kappa}^{T} & =\left\{{ }^{(0)} \kappa_{x x},{ }^{(0)} \kappa_{y y},{ }^{(0)} \kappa_{z z},{ }^{(0)} \kappa_{x y},{ }^{(1)} \kappa_{x x},{ }^{(1)} \kappa_{y y},{ }^{(1)} \kappa_{x y}\right\}
\end{aligned}
$$

\section{Governing Equations}

The governing equations concerning the equilibrium equations and continuity of interelement displacements along the element edges are derived based on the principle of minimum potential energy. The unknown traction components, $\lambda_{x}, \lambda_{y}$, and $\lambda_{z}$, acting along the element edge ensure the continuity of interelement displacements.

For a sandwich element subjected to normal tractions on the top and bottom surfaces, $q^{+}(x, y, h)$ and $q^{-}(x, y,-h)$, respectively, and the unknown interelement tractions, $\lambda_{x}, \lambda_{y}$, and $\lambda_{z}$, along the edges, the total potential energy, $\Pi_{e}$, of the element " $e$ " is expressed as 


$$
\begin{aligned}
\Pi_{e} & =\int_{A_{e}} \int_{-h}^{h}\left(\sigma_{x x}^{(k)} \varepsilon_{x x}+\sigma_{y y}^{(k)} \varepsilon_{y y}+\sigma_{z z} \varepsilon_{z z}^{(k)}+\sigma_{y z}^{(k)} \gamma_{y z}\right. \\
& \left.+\sigma_{x z}^{(k)} \gamma_{x z}+\sigma_{x y}^{(k)} \gamma_{x y}\right) d z d A-\int_{A_{e}}\left(q^{+} u_{z}(x, y, h)\right) d A \\
& +\int_{A_{e}}\left(q^{-} u_{z}(x, y,-h)\right) d A \\
& -\int_{\Gamma_{e}} \int_{-h}^{h}\left[\lambda_{x}\left(u_{x}-u_{x a}\right)+\lambda_{y}\left(u_{y}-u_{y a}\right)\right. \\
& \left.+\lambda_{z}\left(u_{z}-u_{z a}\right)\right] d z d \Gamma
\end{aligned}
$$

where $A_{e}$ represents the area of the element mid-surface and $\Gamma_{e}$ the boundary of the element. The displacement components, $u_{x a}, u_{v a}$, and $u_{z a}$, belong to the adjacent elements denoted by $a$. In accordance with the $\{3,2\}$ plate theory, the weighted-average quantities associated with the adjacent element are represented by $u_{a}, v_{a}, w_{a}, \theta_{x a}$, and $\theta_{y a}$.

The governing equations are obtained by enforcing the first variation of the total potential energy with respect to the element unknowns (i.e., the displacement components, $u_{x}, u_{y}$, and $u_{z}$, and the interelement tractions, $\lambda_{x}, \lambda_{y}$, and $\lambda_{z}$ ) to vanish. This requirement results in

$$
\begin{aligned}
& \delta \Pi_{e}=\int_{A_{e}-h}^{h} \int_{-h}^{h}\left(\sigma_{x x}^{(k)} \delta \varepsilon_{x x}+\sigma_{y y}^{(k)} \delta \varepsilon_{y y}+\sigma_{z z} \delta \varepsilon_{z z}^{(k)}+\sigma_{y z}^{(k)} \delta \gamma_{y z}\right. \\
& \left.+\sigma_{x z}^{(k)} \delta \gamma_{x z}+\sigma_{x y}^{(k)} \delta \gamma_{x y}+\sigma_{x z}^{(k)} \delta \gamma_{x z}+\sigma_{x y}^{(k)} \delta \gamma_{x y}\right) d z d A \\
& \quad-\int_{A_{e}}\left(q^{+} \delta u_{z}(x, y, h)\right) d A \\
& +\int_{A_{e}}\left(q^{-} \delta u_{z}(x, y,-h)\right) d A \\
& -\int_{\Gamma_{e}-h}^{h} \int_{-h}^{h}\left[\delta \lambda_{x}\left(u_{x}-u_{x a}\right)+\delta \lambda_{y}\left(u_{y}-u_{y a}\right)\right. \\
& \left.+\delta \lambda_{z}\left(u_{z}-u_{z a}\right)\right] d z d \Gamma \\
& -\int_{\Gamma_{e}}^{h} \int_{-h}^{h}\left[\lambda_{x} \delta u_{x}+\lambda_{y} \delta u_{y}+\lambda_{z} \delta u_{z}\right] d z d \Gamma=0
\end{aligned}
$$

Substituting for the displacement and strain components from Eqs. (1), (3), (4), and (10) in conjunction with Eqs. (11) and (12) and integrating through the thickness in Eq. (17) result in

$$
\begin{aligned}
\delta \Pi_{e} & =\int_{4}\left\{{ }^{(0)} N_{x x} \delta^{(0)} \varepsilon_{x x}+{ }^{(0)} N_{y y} \delta^{(0)} \varepsilon_{y y}+{ }^{(0)} N_{z z} \delta^{(0)} \varepsilon_{z z}+{ }^{(0)} N_{x y} \delta^{(0)} \gamma_{x y}\right. \\
& +{ }^{(1)} N_{x x} \delta^{(1)} \varepsilon_{x x}+{ }^{(1)} N_{y y} \delta^{(1)} \varepsilon_{y y}+{ }^{(1)} N_{x y} \delta^{(1)} \gamma_{x y} \\
& +{ }^{(0)} M_{x x} \delta^{(0)} \kappa_{x x}+{ }^{(0)} M_{y y} \delta^{(0)} \kappa_{y y}+{ }^{(0)} M_{z z} \delta^{(0)} \kappa_{z z}+{ }^{(0)} M_{x y} \delta^{(0)} \kappa_{x y}
\end{aligned}
$$

(continued in next column)

$$
\begin{aligned}
& +{ }^{(1)} M_{x x} \delta^{(1)} \kappa_{x x}+{ }^{(1)} M_{y y} \delta^{(1)} \kappa_{y y}+{ }^{(1)} M_{x y} \delta^{(1)} \kappa_{x y} \\
& \left.+{ }^{(0)} Q_{y z} \delta^{(0)} \gamma_{y z}+{ }^{(0)} Q_{x z} \delta^{(0)} \gamma_{x z}\right\} d A \\
& +\int_{A}\left\{\left(q^{+}-q^{-}\right) \delta w+\left(q^{+}-q^{-}\right) \delta w_{1}+\frac{4}{5}\left(q^{+}-q^{-}\right) \delta w_{2}\right\} d A \\
& +\int_{\Gamma_{y}}\left\{\delta^{(0)} \hat{T}_{x}\left(u-u_{a}\right)+\delta^{(0)} \hat{M}_{x}\left(\theta_{y}-\theta_{y a}\right)+\delta^{(1)} \hat{T}_{x} h\left(w_{1, x}-w_{1, x a}\right)\right. \\
& +\delta^{(1)} \hat{M}_{x}\left[\left(\frac{5}{4}\left(\theta_{y}+w_{x, x}\right)+w_{2, x}\right)-\left(\frac{5}{4}\left(\theta_{y a}+w_{a, x}\right)+w_{2 a, x}\right)\right] \\
& +\delta^{(0)} \hat{T}_{y}\left(v-v_{a}\right)+\delta^{(0)} \hat{M}_{x}\left(\theta_{x}-\theta_{x a}\right)+\delta^{(1)} \hat{T}_{y} h\left(w_{1, y}-w_{1, y a}\right) \\
& +\delta^{(1)} \hat{M}_{y}\left[\left(\frac{5}{4}\left(\theta_{x}+w_{, y}\right)+w_{2, y}\right)-\left(\frac{5}{4}\left(\theta_{x a}+w_{a, y}\right)+w_{2 a, y}\right)\right] \\
& \left.+\delta^{(0)} \hat{Q}_{n z}\left(w-w_{a}\right)+\delta^{(1)} \hat{Q}_{n z}\left(w_{1}-w_{1 a}\right)+\delta^{(2)} \hat{Q}_{n z}\left(w_{2}-w_{2 a}\right)\right\} d \Gamma \\
& +\int_{\Gamma_{r}}\left\{{ }^{(1)} \hat{M}_{x} \delta\left(\frac{5}{4}\left(\theta_{y}+w_{x x}\right)+w_{2, x}\right)\right. \\
& +{ }^{(0)} \hat{T}_{y} \delta v+{ }^{(0)} \hat{M}_{x} \delta \theta_{x}+{ }^{(1)} \hat{T}_{y} h \delta w_{1, y} \\
& +{ }^{(1)} \hat{M}_{y} \delta\left(\frac{5}{4}\left(\theta_{x}+w_{, y}\right)+w_{2, y}\right) \\
& \left.+{ }^{(0)} \hat{Q}_{n z} \delta w+{ }^{(1)} \hat{Q}_{n z} \delta w_{1}+{ }^{(2)} \hat{Q}_{n z} \delta w_{2}\right\} d \Gamma=0
\end{aligned}
$$

where the left superscripts (0) and (1) denote the constant- and higher-order modes of deformations in the transverse direction, respectively. The expressions for the interelement stress and moment resultants, ${ }^{(i)} \hat{T}_{\alpha}$, ${ }^{(i)} \hat{Q}_{n z}$, and ${ }^{(i)} \hat{M}_{\alpha} \quad(\alpha=x, y ; i=0,1,2)$, are defined as

$$
\begin{aligned}
& \left({ }^{(0)} \hat{T}_{x},{ }^{(0)} \hat{M}_{x},{ }^{(1)} \hat{T}_{x},{ }^{(1)} \hat{M}_{x}\right)=\int_{-h}^{h} \lambda_{x}\left(P_{0}, P_{1}, P_{2}, P_{3}\right) d z \\
& \left({ }^{(0)} \hat{T}_{y},{ }^{(0)} \hat{M}_{y},{ }^{(1)} \hat{T}_{y},{ }^{(1)} \hat{M}_{y}\right)=\int_{-h}^{h} \lambda_{y}\left(P_{0}, P_{1}, P_{2}, P_{3}\right) d z \\
& \left({ }^{(0)} \hat{Q}_{n z},{ }^{(1)} \hat{Q}_{n z},{ }^{(2)} \hat{Q}_{n z}\right)=\int_{-h}^{h} \lambda_{z}\left(1, \xi, \xi^{2}-\frac{1}{5}\right) d z
\end{aligned}
$$

in which

$$
\begin{aligned}
& P_{0}=1, \mathrm{P}_{1}=h \xi, P_{2}=\left(\frac{1}{6}-\frac{\xi^{2}}{2}\right) \\
& P_{3}=h\left(\frac{\xi}{5}-\frac{\xi^{3}}{3}\right)
\end{aligned}
$$

The resultant stresses, ${ }^{(\ell)} N_{\alpha \beta},{ }^{(0)} Q_{y z}$, and ${ }^{(0)} Q_{x z}$, and the resultant moments, ${ }^{(\ell)} M_{\alpha \beta}(\alpha, \beta=x, y ; \quad \ell=0,1)$, can be expressed in terms of the strain and curvature components by integrating the strain energy density of the sandwich panel [i.e., the first integrand in Eq. (16)] 
through the thickness after substituting from Eqs. (4), (6), (10), and (11) in conjunction with Eq. (13) as

$$
\left\{\begin{array}{l}
\mathbf{N} \\
\mathbf{M} \\
\mathbf{Q}
\end{array}\right\}=\left[\begin{array}{ccc}
\mathbf{A} & \mathbf{B} & \mathbf{0} \\
\mathbf{B}^{T} & \mathbf{D} & \mathbf{0} \\
\mathbf{0} & \mathbf{0} & \mathbf{G}
\end{array}\right]\left\{\begin{array}{l}
\mathbf{o} \\
\mathbf{\kappa} \\
\boldsymbol{\gamma}
\end{array}\right\}-\left\{\begin{array}{c}
\mathbf{N}^{*} \\
\mathbf{M}^{*} \\
\mathbf{0}
\end{array}\right\} T(x, y)
$$

where

$$
\begin{aligned}
& \mathbf{N}^{T}=\left\{{ }^{(0)} N_{x x},{ }^{(0)} N_{y y},{ }^{(0)} N_{z z},{ }^{(0)} N_{x y},{ }^{(1)} N_{x x},{ }^{(1)} N_{y y},{ }^{(1)} N_{x y}\right\} \\
& \mathbf{M}^{T}=\left\{{ }^{(0)} M_{x x},{ }^{(0)} M_{y y},{ }^{(0)} M_{z z},{ }^{(0)} M_{x y},{ }^{(1)} M_{x x},{ }^{(1)} M_{y y},{ }^{(1)} M_{x y}\right\} \\
& \mathbf{N}^{*}=\left\{{ }^{(0)} N_{x x}^{*},{ }^{(0)} N_{y y}^{*},{ }^{(0)} N_{z z}^{*},{ }^{(0)} N_{x y}^{*},{ }^{(1)} N_{x x}^{*},{ }^{(1)} N_{y y}^{*},{ }^{(1)} N_{x y}^{*}\right\}^{T} \\
& \mathbf{M}^{*}=\left\{{ }^{(0)} M_{x x}^{*},{ }^{(0)} M_{y y}^{*},{ }^{(0)} M_{z z}^{*},{ }^{(0)} M_{x y}^{*},{ }^{(1)} M_{x x}^{*},{ }^{(1)} M_{y y}^{*},{ }^{(1)} M_{x y}^{*}\right\}^{T} \\
& \mathbf{Q}^{T}=\left\{{ }^{(0)} Q_{y z},{ }^{(0)} Q_{x z}\right\} \\
& \text { ò } \mathbf{E}=\left\{{ }^{(0)} \varepsilon_{x x},{ }^{(0)} \varepsilon_{y y},{ }^{(0)} \varepsilon_{z z},{ }^{(0)} \gamma_{x y},{ }^{(1)} \varepsilon_{x x},{ }^{(1)} \varepsilon_{y y},{ }^{(1)} \gamma_{x y}\right\} \\
& \boldsymbol{\kappa}^{T}=\left\{{ }^{(0)} \kappa_{x x},{ }^{(0)} \kappa_{y y},{ }^{(0)} \kappa_{z z},{ }^{(0)} \kappa_{x y},{ }^{(1)} \kappa_{x x},{ }^{(1)} \kappa_{y y},{ }^{(1)} \kappa_{x y}\right\}(21 \mathrm{~g}) \\
& \boldsymbol{\gamma}^{T}=\left\{{ }^{(0)} \gamma_{y z},{ }^{(0)} \gamma_{x z}\right\}
\end{aligned}
$$

The explicit forms of $\mathbf{A}, \mathbf{B}, \mathbf{D}, \mathbf{N}^{*}$, and $\mathbf{M}^{*}$ are given in Appendix B. This constitutive relation can be also expressed in terms of the compliance matrix $\mathbf{C}$ in the form

where

$$
\mathbf{E}=\mathbf{C}\left(\mathbf{S}+\mathbf{S}_{t}\right)
$$

$$
\begin{aligned}
& \mathbf{E}^{T}=\{\grave{\mathbf{o}}, \kappa, \boldsymbol{\gamma}\}, \quad \mathbf{S}^{T}=\{\mathbf{N}, \mathbf{M}, \mathbf{Q}\} \\
& \mathbf{S}_{t}^{T}=T(x, y)\left\{\mathbf{N}^{*}, \mathbf{M}^{*}, \mathbf{0}\right\}
\end{aligned}
$$

Substituting for the resultant strains and curvatures from Eq. (5), integrating the area integrals of Eq. (18) by parts, and applying the Gauss theorem to appropriate terms result in the governing equations for the sandwich element. The equations of equilibrium are obtained as

$$
\begin{gathered}
{ }^{(0)} N_{x x, x}+{ }^{(0)} N_{x y, y}=0 \\
{ }^{(0)} N_{y y, y}+{ }^{(0)} N_{x y, x}=0 \\
\frac{5}{4}\left({ }^{(1)} M_{x x, x x}+{ }^{(1)} M_{y y, y y}+2{ }^{(1)} M_{x y, x y}\right) \\
-{ }^{(0)} Q_{x z, x}-{ }^{(0)} Q_{y z, y}=\bar{q}_{1}
\end{gathered}
$$

$$
\begin{gathered}
\frac{5}{4}\left({ }^{(1)} M_{y y, y}+{ }^{(1)} M_{x y, x}\right)+{ }^{(0)} M_{y y, y} \\
+{ }^{(0)} M_{x y, x}-{ }^{(0)} Q_{y z}=0 \\
\frac{5}{4}\left({ }^{(1)} M_{x x, x}+{ }^{(1)} M_{x y, y}\right)+{ }^{(0)} M_{x x, x} \\
+{ }^{(0)} M_{x y, y}-{ }^{(0)} Q_{x z}=0 \\
\frac{{ }^{(0)} N_{z z}}{h}+h\left({ }^{(1)} N_{x x, x x}+{ }^{(1)} N_{y y, y y}+2^{(1)} N_{x y, x y}\right)=\bar{q}_{2} \\
\frac{{ }^{(0)} M_{z z}}{h^{2}}+\left({ }^{(1)} M_{x x, x x}+{ }^{(1)} M_{y y, y y}+2^{(1)} M_{x y, x y}\right)=\frac{4}{5} \bar{q}_{1}
\end{gathered}
$$

where

$$
\bar{q}_{1}=q^{+}-q^{-} \text {and } \bar{q}_{2}=q^{+}+q^{-}
$$

The interelement stress and moment resultants, ${ }^{(i)} \hat{T}_{\alpha}$, ${ }^{(i)} \hat{Q}_{n z}$, and ${ }^{(i)} \hat{M}_{\alpha} \quad(\alpha=x, y ; i=0,1,2)$, along the element boundaries are obtained as

$$
\begin{gathered}
{ }^{(0)} \hat{T}_{x}={ }^{(0)} N_{x x} n_{x}+{ }^{(0)} N_{x y} n_{y} \\
{ }^{(0)} \hat{T}_{y}={ }^{(0)} N_{y y} n_{y}+{ }^{(0)} N_{x y} n_{x} \\
{ }^{(0)} \hat{Q}_{n z}={ }^{(0)} Q_{x z} n_{x}+{ }^{(0)} Q_{y z} n_{y} \\
-\frac{5}{4}\left({ }^{(1)} M_{x x, x} n_{x}+{ }^{(1)} M_{x y, x} n_{y}\right) \\
-\frac{5}{4}\left({ }^{(1)} M_{y y, y} n_{y}+{ }^{(1)} M_{x y, y} n_{x}\right) \\
{ }^{(0)} \hat{M}_{y}={ }^{(0)} M_{y y} n_{y}+{ }^{(0)} M_{x y} n_{x} \\
{ }^{(0)} \hat{M}_{x}={ }^{(0)} M_{x x} n_{x}+{ }^{(0)} M_{x y} n_{y} \\
{ }^{(1)} \hat{Q}_{n z}=-h\left({ }^{(1)} N_{x x, x} n_{x}+{ }^{(1)} N_{y y, y} n_{y}\right. \\
\left.+{ }^{(1)} N_{x y, x} n_{y}+{ }^{(1)} N_{x y, y} n_{x}\right) \\
{ }^{(1)} \hat{T}_{x}={ }^{(1)} N_{x x} n_{x}+{ }^{(1)} N_{x y} n_{y} \\
{ }^{(1)} \hat{T}_{y}={ }^{(1)} N_{y y} n_{y}+{ }^{(1)} N_{x y} n_{x} \\
\hat{M}_{x}={ }^{(1)} M_{x y} n_{y} n_{y}+{ }^{(1)} M_{x y} n_{y} \\
+\left({ }^{(1)} M_{x x, x} n_{x}+{ }^{(1)} M_{x y, x} n_{y}\right. \\
\left.+{ }^{(1)} M_{y y, y} n_{y}+{ }^{(1)} M_{x y, y} n_{x}\right) \\
{ }^{(1)}{ }^{(1)}
\end{gathered}
$$


The interelement kinematic continuity conditions are obtained as

$$
\begin{aligned}
& u=u_{a}, v=v_{a}, w=w_{a} ; \theta_{x}=\theta_{x a}, \theta_{y}=\theta_{y a} \\
& w_{1}=w_{1 a}, w_{1, x}=w_{1 a, x}, w_{1, y}=w_{1 a, y}, w_{2}=w_{2 a} \\
& {\left[\frac{5}{4}\left(\theta_{y}+w_{, x}\right)+w_{2, x}\right]=\left[\frac{5}{4}\left(\theta_{y a}+w_{a, x}\right)+w_{2 a, x}\right]} \\
& {\left[\frac{5}{4}\left(\theta_{x}+w_{, y}\right)+w_{2, y}\right]=\left[\frac{5}{4}\left(\theta_{x a}+w_{a, y}\right)+w_{2 a, y}\right]}
\end{aligned}
$$

These kinematic continuity conditions are imposed not only on the weighted-average displacements and slopes $\left(u, v, w, \theta_{x}, \theta_{y}\right)$, but also on the derivatives of the higher-order displacement modes $\left(w_{1}, w_{2}\right)$ in the transverse direction. Therefore, the finite element implementation of the equilibrium equations requires at least $C^{1}$ interelement continuity for the out-of-plane displacement modes of $w, w_{1}$, and $w_{2}$. Because of this requirement, the finite element implementation of the total potential energy functional in terms of the assumed displacement field becomes rather difficult. Therefore, this triangular sandwich element is developed by utilizing the hybrid energy functional in which the stress/ moment resultants within the element and displacements and slopes around the element boundaries are assumed independently.

\section{Element Development}

The hybrid energy functional formulation overcomes the difficulty of the $C^{1}$ interelement continuity requirement because the displacements, as well as the slopes, are independently assumed only along element boundaries, which can be rendered identical along the common boundaries of adjacent elements. However, the kinematic compatibility between the displacements and slopes along the element boundaries is preserved in order to avoid a possible shear-locking phenomenon. Also, as part of the hybrid energy functional formulation, the stress and moment resultants within the element are selected in the form of complex power series so as to satisfy the equilibrium equations of Eq. (24).

The hybrid energy functional for an element, $\Pi_{H}$, is defined as

$$
\Pi_{H}=\frac{1}{2} \int_{A_{e}} \mathbf{S}^{T} \mathbf{C S} d A+\int_{A_{e}} \mathbf{S}_{t}^{T} \mathbf{E} d A-\int_{\Gamma_{e}} \mathbf{T}_{b}^{T} \mathbf{u}_{b} d \Gamma
$$

in which the element boundary is denoted by $\Gamma_{e}$ and its area by $A_{e}$. The vectors $\mathbf{T}_{b}$ and $\mathbf{u}_{b}$ include the components of the boundary forces and boundary displacements, respectively. The compliance matrix, $\mathbf{C}$, is defined in Eq. (22). In accordance with the hybrid energy formulation, the resultant stress vector, $\mathbf{S}$, must satisfy the equilibrium equations, Eq. (24), identically. It is composed of the homogeneous solution, $\mathbf{S}_{h}$, and the particular solution, $\mathbf{S}_{o}$, in the form

$$
\mathbf{S}=\mathbf{S}_{h}+\mathbf{S}_{o}
$$

The components of the homogeneous part of the resultant stress vector, $\mathbf{S}_{h}$, are expressed in the form of complex power series as

$$
\begin{aligned}
& { }^{(0)} N_{\xi \eta}=\sum_{k=0}^{4}{ }^{(0)} \mathbf{N}_{\xi \eta(k)}^{T}{ }^{(0)} \boldsymbol{\alpha}_{\xi \eta(k)} \\
& { }^{(1)} N_{\xi \eta}=\sum_{k=0}^{4}{ }^{(1)} \mathbf{N}_{\xi \eta(k)}^{T}{ }^{(1)} \boldsymbol{\alpha}_{\xi \eta(k)} \\
& { }^{(0)} N_{z z}=\sum_{k=0}^{2}{ }^{(0)} \mathbf{N}_{z z(k)}^{T}{ }^{(0)} \boldsymbol{\alpha}_{z z(k)} \\
& { }^{(0)} M_{\xi \eta}=\sum_{k=0}^{4}{ }^{\left({ }^{(0)}\right.} \mathbf{M}_{\xi \eta(k)}^{T}{ }^{(0)} \boldsymbol{\beta}_{\xi \eta(k)} \\
& { }^{(1)} M_{\xi \eta}=\sum_{k=0}^{4}{ }^{(1)} \mathbf{M}_{\xi \eta(k)}^{T}{ }^{(1)} \boldsymbol{\beta}_{\xi \eta(k)} \\
& { }^{(0)} M_{z z}=\sum_{k=0}^{2}{ }^{(0)} \mathbf{M}_{z z(k)}^{T}{ }^{(0)} \boldsymbol{\beta}_{z z(k)} \\
& { }^{(0)} Q_{\xi z}=\sum_{k=0}^{3}{ }^{\left({ }^{(0)}\right.} \mathbf{Q}_{\xi z(k)}^{T}{ }^{(0)} \boldsymbol{\beta}_{\xi z(k)}
\end{aligned}
$$

in which the vectors of stress, ${ }^{(\ell)} \mathbf{N}_{\xi \eta}$, moment, ${ }^{(\ell)} \mathbf{M}_{\xi \eta}$, and shear stress, ${ }^{(0)} \mathbf{Q}_{\xi z}$, resultants contain the real and imaginary parts of the complex power series terms, and the vectors ${ }^{(\ell)} \boldsymbol{\alpha}_{\xi \eta(k)},{ }^{(\ell)} \boldsymbol{\beta}_{\xi \eta(k)}$, and ${ }^{(0)} \boldsymbol{\rho}_{\xi z(k)}$ contain the real and imaginary parts of the unknown coefficients (generalized coordinates) with $\xi, \eta=x, y ; \ell=0,1$. The series representations are truncated at an appropriate order so as to ensure compatibility among the stress, moment, and shear stress resultants, when substituted into the equilibrium equations, and to ensure definiteness of the stiffness matrix associated with the generalized coordinates. With this representation, the homogeneous equilibrium equations are satisfied, provided that certain relations exist among the unknown coefficients. These constraint conditions are expressed as

$$
\mathbf{c b}=\mathbf{0}
$$

in which $\mathbf{c}$ is the constraint matrix and $\mathbf{b}$ is the vector of unknown coefficients. The constraint equations that are required to construct matrix $\mathbf{c}$ are given in Appen- 
dix C. The vectors ${ }^{(\ell)} \mathbf{N}_{\xi \eta(k)},{ }^{(\ell)} \mathbf{M}_{\xi \eta(k)}$, and ${ }^{(0)} \mathbf{Q}_{\xi z(k)}$, and their associated unknown coefficient vectors, ${ }^{(\ell)} \boldsymbol{\alpha}_{\xi \eta(k)},{ }^{(\ell)} \boldsymbol{\beta}_{\xi \eta(k)}$, and ${ }^{(0)} \boldsymbol{\rho}_{\xi z(k)}$, are expressed in the form

$$
\begin{aligned}
& { }^{(\ell)} \mathbf{N}_{\xi \eta(k)}^{T}={ }^{(i)} \mathbf{M}_{\xi \eta(k)}^{T}=\left\{\operatorname{Re}\left[z^{k}\right],-\operatorname{Im}\left[z^{k}\right]\right\} \\
& (k=0,1,2,3,4) \\
& { }^{(\ell)} \mathbf{N}_{z z(k)}^{T}={ }^{(\ell)} \mathbf{M}_{z z(k)}^{T}=\left\{\operatorname{Re}\left[z^{k}\right],-\operatorname{Im}\left[z^{k}\right]\right\} \\
& (k=0,1,2) \\
& { }^{(0)} \mathbf{Q}_{\xi z(k)}^{T}=\left\{\operatorname{Re}\left[z^{k}\right],-\operatorname{Im}\left[z^{k}\right]\right\} \\
& (k=0,1,2,3) \\
& { }^{(\ell)} \boldsymbol{\alpha}_{\xi \eta(k)}^{T}=\left\{\operatorname{Re}\left[{ }^{(\ell)} \alpha_{\xi \eta(k)}\right], \operatorname{Im}\left[{ }^{(\ell)} \alpha_{\xi \eta(k)}\right]\right\} \\
& (k=0,1,2,3,4) \\
& { }^{(\ell)} \boldsymbol{\alpha}_{z z(k)}^{T}=\left\{\operatorname{Re}\left[{ }^{(\ell)} \alpha_{z z(k)}\right], \operatorname{Im}\left[{ }^{(\ell)} \alpha_{z z(k)}\right]\right\} \\
& (k=0,1,2) \\
& { }^{(\ell)} \boldsymbol{\beta}_{\xi \eta(k)}^{T}=\left\{\operatorname{Re}\left[{ }^{(\ell)} \beta_{\xi \eta(k)}\right], \operatorname{Im}\left[{ }^{(\ell)} \beta_{\xi \eta(k)}\right]\right\} \\
& (k=0,1,2,3,4) \\
& { }^{(\ell)} \beta_{z z(k)}^{T}=\left\{\operatorname{Re}\left[{ }^{(\ell)} \beta_{z z(k)}\right], \operatorname{Im}\left[{ }^{(\ell)} \beta_{z z(k)}\right]\right\} \\
& (k=0,1,2) \\
& { }^{(0)} \rho_{\xi z(k)}^{T}=\left\{\operatorname{Re}\left[{ }^{(0)} \rho_{\xi z(k)}\right], \operatorname{Im}\left[{ }^{(0)} \rho_{\xi z(k)}\right]\right\} \\
& (k=0,1,2,3)
\end{aligned}
$$

with $\xi, \eta=x, y$ and $\ell=0,1$; the complex variable, $z$, is defined as $z=x+i y$, in which $i=\sqrt{-1}$. The vector representations of the stress, bending, and shear stress resultants can be cast into matrix form as

$$
\mathbf{S}_{h}=\mathbf{P b}
$$

in which the matrix $\mathbf{P}$ contains the real and imaginary parts of the power series terms and the vector $\mathbf{b}$ contains the real and imaginary parts of all the unknown generalized coordinates. The explicit expressions for $\mathbf{P}$ and $\mathbf{b}$ are given in Appendix C. Hence, the general solution form of the resultant stress vector $\mathbf{S}$ becomes

$$
\mathbf{S}=\mathbf{P b}+\mathbf{S}_{o}
$$

As part of the hybrid finite element formulation, the displacement field is assumed along the element boundary. Along the $k^{\text {th }}$ edge of the triangular element lying between nodes $i$ and $j$ (as shown in Fig. 2), a Cartesian coordinate system is attached to node $i$ and the axes normal and parallel to the $k^{\text {th }}$ edge are referred to as $n$ and $s$, respectively. The $s$-axis also represents the line coordinate along the boundary, and it varies be-

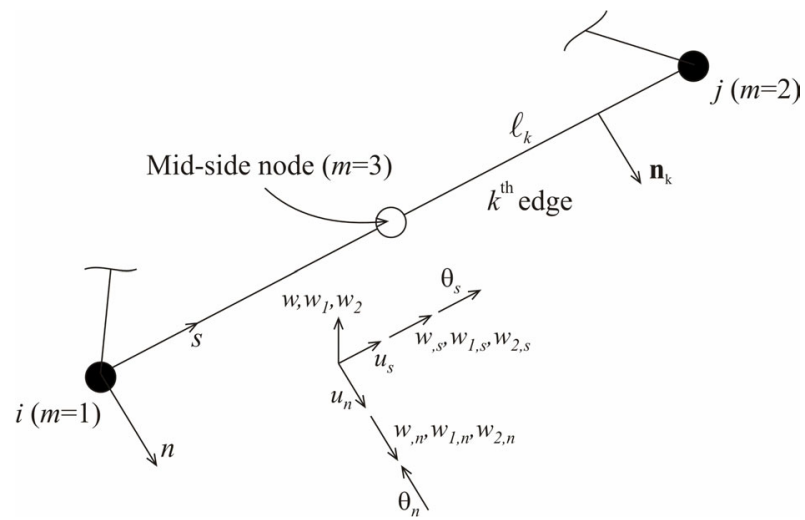

Fig. 2 Assumed displacement and slope fields along the element boundary.

tween $0 \leq s \leq \ell_{k}$, with $\ell_{k}$ being the length of the $k^{\text {th }}$ edge. Therefore, the boundary displacements and their derivatives, as well as the independent slope components, as defined by the $\{3,2\}$-order theory, are expressed in terms of the nodal degrees of freedom as

$$
\begin{gathered}
\left(u_{n}^{(k)}, u_{s}^{(k)}\right)=\sum_{m=1}^{2} N_{m}(s)\left(u_{n(m)}^{(k)}, u_{s(m)}^{(k)}\right) \\
\left(w^{(k)}, w_{1}^{(k)}, w_{2}^{(k)}\right)=\sum_{m=1}^{2} H_{m}(s)\left(w_{(m)}^{(k)}, w_{1(m)}^{(k)}, w_{2(m)}^{(k)}\right) \\
+H_{m+2}(s)\left(w_{, s(m)}^{(k)}, w_{1, s(m)}^{(k)}, w_{2, s(m)}^{(k)}\right) \\
\left(w_{, n}^{(k)}, w_{1, n}^{(k)}, w_{2, n}^{(k)}\right)=\sum_{m=1}^{2} N_{m}(s)\left(w_{, n(m)}^{(k)}, w_{1, n(m)}^{(k)}, w_{2, n(m)}^{(k)}\right) \\
\theta_{s}^{(k)}=\sum_{m=1}^{2} N_{m}(s) \theta_{s(m)}^{(k)} \\
\theta_{n}^{(k)}=\sum_{m=1}^{3} M_{m}(s) \theta_{n(m)}^{(k)}
\end{gathered}
$$

where $\left(u_{n}^{(k)}, u_{s}^{(k)}\right)$ represents the in-plane displacements normal and tangent to the $k^{\text {th }}$ edge and $\left(w^{(k)}, w_{1}^{(k)}, w_{2}^{(k)}\right)$ and $\left(w_{, n}^{(k)}, w_{1, n}^{(k)}, w_{2, n}^{(k)}\right)$ denote the weighted-average, first-, and second-order modes of out-of-plane displacements and their derivatives, respectively. The slopes normal and tangent to the boundary are denoted by $\theta_{n}^{(k)}$ and $\theta_{s}^{(k)}$, respectively, and their sign conventions are shown in Fig. 2. The corresponding nodal degrees of freedom for these displacement and slope components at nodes $i$ and $j$ along the $k^{\text {th }}$ edge are identified by the subscript $m=1$ and $m=2$, respectively. Note that the slope component, $\theta_{s}^{(k)}$, has a mid-side (third-node) degree of freedom to be eliminated later.

The functions $N_{j}(s), M_{j}(s)$, and $H_{j}(s)$ represent the linear, quadratic, and cubic (Hermitian) interpolation functions, respectively. Also, the out-of-plane dis- 
placement components, $w^{(k)}, w_{1}^{(k)}$, and $w_{2}^{(k)}$, are functions of the $s$-axis only. Therefore, the derivatives of $\left(w^{(k)}, w_{1}^{(k)}, w_{2}^{(k)}\right)$ with respect to the $n$-axis are assumed independently by using linear shape functions.

In order to maintain kinematic compatibility, different orders of polynomials are chosen to represent the displacement and slope components as given in Eq. (35). The degree of freedom at the mid-side node, $\theta_{n(3)}^{(k)}$, is eliminated by imposing the constraint of linear variation of the boundary expression

$$
\frac{\partial^{2}}{\partial s^{2}}\left[\frac{5}{4}\left(\theta_{n}^{(k)}+w_{, s}^{(k)}\right)+w_{2, s}^{(k)}\right]=0
$$

With this constraint equation, the mid-side degree of freedom, $\theta_{n(3)}^{(k)}$, can be expressed in terms of $\theta_{n(m)}^{(k)}$, $w_{, s(m)}^{(k)}$, and $w_{2, s}^{(k)}$, with $(m=1,2)$, which are assigned at the ends of the $k^{\text {th }}$ edge.

In compact form, the in-plane displacements, $u_{n}^{(k)}$ and $u_{s}^{(k)}$, the transverse displacements, $w^{(k)}, w_{1}^{(k)}$, and $w_{2}^{(k)}$, their derivatives with respect to the $n$ - and $s$ coordinates, and the slopes around the $n$ - and $s$-axes, $\theta_{n}^{(k)}$ and $\theta_{s}^{(k)}$, along the $k^{\text {th }}$ edge of the element can be expressed as

$$
\mathbf{u}^{\prime(k)}=\mathbf{L}^{\prime(k)} \mathbf{v}^{\prime(k)}
$$

where

$$
\begin{aligned}
\mathbf{u}^{\prime(k)^{T}}= & \left\{u_{n}^{(k)}, u_{s}^{(k)}, w^{(k)}, w_{1}^{(k)}, w_{2}^{(k)}, w_{, n}^{(k)}, w_{1, n}^{(k)}, w_{2, n}^{(k)},\right. \\
& \left.w_{, s}^{(k)}, w_{1, s}^{(k)}, w_{2, s}^{(k)}, \theta_{n}^{(k)}, \theta_{s}^{(k)}\right\} \\
\mathbf{v}^{\prime(k)^{T}}= & \left\{\mathbf{u}_{n}^{\prime(k)^{T}}, \mathbf{u}_{s}^{\prime(k)^{T}}, \mathbf{w}^{\prime(k)^{T}}, \mathbf{w}_{1}^{\prime(k)^{T}}, \mathbf{w}_{2}^{\prime(k)^{T}},\right. \\
& \mathbf{w}_{, n}^{\prime(k)^{T}}, \mathbf{w}_{1, n}^{\prime(k)^{T}}, \mathbf{w}_{2, n}^{\prime(k)^{T}}, \mathbf{w}_{, s}^{\prime(k)^{T}}, \\
& \left.\mathbf{w}_{1, s}^{\prime(k)^{T}}, \mathbf{w}_{1, s}^{\prime(k)}, \mathbf{w}_{2, s}^{\prime(k)^{T}}, \boldsymbol{\theta}_{n}^{\prime(k)^{T}}, \boldsymbol{\theta}_{s}^{\prime(k)^{T}}\right\}
\end{aligned}
$$

in which $\mathbf{u}_{\alpha}^{\prime(k)^{T}}=\left\{u_{\alpha(1)}^{(k)}, u_{\alpha(\mathcal{F})}^{(k)}\right\}, \quad \mathbf{w}^{\prime(k)^{T}}=\left\{w_{(1)}^{(k)}, w_{(\mathcal{p})}^{(k)}\right\}$, $\mathbf{w}_{, \alpha}^{\prime(k)^{T}}=\left\{w_{, \alpha(1)}^{(k)}, w_{, \alpha(2)}^{(k)}\right\} ; \theta_{\alpha}^{\prime(k)}=\left\{\theta_{\alpha(1)}^{(k)}, \theta_{\alpha(2)}^{(k)}\right\}, \quad \mathbf{w}_{i}^{\prime(k)^{\prime}}=$ $\left\{w_{i(1)}^{(k)}, w_{i(2)}^{(k)}\right\}$, and $\mathbf{w}_{i, \alpha}^{\prime(k)}=\left\{w_{i, \alpha(1)}^{(k)}, w_{i, \alpha(2)}^{(k)}\right\}$, with $\quad(i=$ $1,2 ; \alpha=n, s)$. The explicit form of the matrix of interpolation functions, $\mathbf{L}^{\prime(k)}$, is given in Appendix C. The prime on the superscript denotes definition with respect to the line coordinate system, $(n, s)$, along the boundary of the element.

In order to utilize this equation in the hybrid energy functional, the vector $\mathbf{v}^{\prime(k)}$ is expressed in terms of the element nodal vector $\mathbf{v}$ as

$$
\mathbf{v}^{\prime(k)}=\mathbf{Z}^{(k)} \mathbf{v}
$$

where $\mathbf{Z}^{(k)}$ is the transformation matrix between the nodal degrees of freedom defined in the element, $(x, y)$, and line coordinate, $(n, s)$, systems for the $k^{\text {th }}$ edge, and $\mathbf{v}^{T}=\left\{\mathbf{v}_{(1)}^{T}, \mathbf{v}_{(2)}^{T}, \mathbf{v}_{(3)}^{T}\right\}$ with

$$
\begin{aligned}
\mathbf{v}_{(k)}^{T}= & \left\{u_{(k)}, v_{(k)}, w_{(k)}, w_{1(k)}, w_{2(k)}, w_{, x(k)}, w_{1, x(k)}, w_{2, x(k)},\right. \\
& \left.w_{2, x(k)}, w_{, y(k)}, w_{1, y(k)}, w_{2, y(k)}, \theta_{x(k)}, \theta_{y(k)}\right\}
\end{aligned}
$$

The form of the transformation matrix, $\mathbf{Z}^{(k)}$, is given explicitly in Appendix C. The displacement vector $\mathbf{u}^{\prime(k)}$ becomes

$$
\mathbf{u}^{\prime(k)}=\mathbf{L}^{\prime(k)} \mathbf{Z}^{(k)} \mathbf{v}
$$

A similar transformation between the displacement vector, $\mathbf{u}^{\prime(k)}$, defined in the line coordinate system and the displacement vector, $\mathbf{u}^{(k)}$, defined in the element coordinate system is established as

where

$$
\mathbf{u}^{\prime(k)}=\mathbf{Y}^{(k)} \mathbf{u}^{(k)}
$$

$$
\begin{aligned}
\mathbf{u}^{(k)}= & \left\{u^{(k)}, v^{(k)}, w^{(k)}, w_{1}^{(k)}, w_{2}^{(k)}, w_{, x}^{(k)}, w_{1, x}^{(k)}, w_{2, x}^{(k)},\right. \\
& \left.w_{, y}^{(k)}, w_{1, y}^{(k)}, w_{2, y}^{(k)}, \theta_{x}^{(k)}, \theta_{y}^{(k)}\right\}
\end{aligned}
$$

and the transformation matrix $\mathbf{Y}^{(k)}$ is expressed in terms of the sine and cosine of the angle between the normal ( $n$-axis) at the $k^{\text {th }}$ edge and the $x$-axis. The explicit expressions for the components of $\mathbf{Y}^{(k)}$ are given in Appendix C. It must be noted that the sign convention of $\theta_{x}^{(k)}$ is in the negative $x$-direction of the element, whereas the slope $\theta_{x(k)}$ at the nodal point is in the positive $x$-direction of the element coordinate system. Also, the transformation matrix is orthonormal, i.e., $\mathbf{Y}^{(k)^{T}}=\mathbf{Y}^{(k)^{-1}}$. Hence, the displacement vector, $\mathbf{u}^{(k)}$, in the element coordinate system is expressed in the terms of the nodal unknowns (or degrees of freedom) in the element coordinate system as

$$
\mathbf{u}^{(k)}=\mathbf{L}^{(k)}(s) \mathbf{v}
$$

in which $\mathbf{L}^{(k)}(s)$ is defined as

$$
\mathbf{L}^{(k)}(s)=\mathbf{Y}^{(k)^{T}} \mathbf{L}^{\prime(k)}(s) \mathbf{Z}^{(k)}
$$

The boundary displacement vector, $\mathbf{u}_{b}^{(k)}$, along the $k^{\text {th }}$ edge of the element is defined as

$$
\begin{aligned}
\mathbf{u}_{b}^{(k)}= & \left\{u^{(k)}, v^{(k)}, w^{(k)}, \theta_{x}^{(k)}, \theta_{y}^{(k)}, w_{1}^{(k)},\right. \\
& w_{1, x}^{(k)}, w_{1, y}^{(k)}, w_{2}^{(k)}, \frac{5}{4}\left(\theta_{y}^{(k)}+w_{, x}^{(k)}\right) \\
+ & \left.w_{2, x}^{(k)}, \frac{5}{4}\left(\theta_{x}^{(k)}+w_{, y}^{(k)}\right)+w_{2, y}^{(k)}\right\}
\end{aligned}
$$

It is related to the displacement vector, $\mathbf{u}^{(k)}$, in the element coordinate system as 


$$
\mathbf{u}_{b}^{(k)}=\mathbf{B}_{\mathbf{u}} \mathbf{u}^{(k)}=\mathbf{B}_{\mathbf{u}} \mathbf{L}^{(k)}(s) \mathbf{v}
$$

in which $\mathbf{B}_{u}$, given explicitly in Appendix $\mathrm{C}$, can easily be constructed by comparing the components of the vectors $\mathbf{u}^{(k)}$ and $\mathbf{u}_{b}^{(k)}$. The boundary stress vector $\mathbf{T}_{b}^{(k)}$, containing the resultant stresses and moments corresponding to the boundary displacement vector $\mathbf{u}_{b}^{(k)}$, can be obtained from

$$
\mathbf{T}_{b}^{(k)}=\mathbf{B}_{s} \mathbf{S}^{(k)}
$$

in which $\mathbf{B}_{s}$ is a matrix transforming the resultant stress vector defined in the element to the resultant stress vector defined along the $k^{\text {th }}$ edge of the element and the explicit form of $\mathbf{B}_{s}$ is given in Appendix C. While invoking the constraint equations, Eq. (30), and substituting for the stress vector, the boundary displacement and boundary stress vectors in the hybrid energy functional result in

$$
\begin{aligned}
\Pi_{H} & =\frac{1}{2} \mathbf{b}^{T} \mathbf{H} \mathbf{b}+\lambda^{T} \mathbf{c b}+{ }^{b} \mathbf{R}_{o}^{T} \mathbf{b}-{ }^{v} \mathbf{R}_{o}^{T} \mathbf{v} \\
& +{ }^{b} \mathbf{R}_{t}^{T} \mathbf{b}-\mathbf{b}^{T} \mathbf{G} \mathbf{v}+\Pi_{o}
\end{aligned}
$$

where

$$
\begin{gathered}
\mathbf{H}=\int_{A_{e}} \mathbf{P}^{T} \mathbf{C} \mathbf{P} d A \\
\mathbf{G}=\sum_{k} \int_{\Gamma_{(k)}} \mathbf{P}^{T} \mathbf{B}_{s}^{(k)} \mathbf{B}_{b}^{(k)} \mathbf{L}^{(k)} d \Gamma \\
{ }^{v} \mathbf{R}_{o}=\sum_{k=1}^{3} \int_{\Gamma_{(k)}} \mathbf{S}_{o}^{T} \mathbf{B}_{s}^{(k)} \mathbf{B}_{b}^{(k)} \mathbf{L}^{(k)} d \Gamma \\
{ }^{b} \mathbf{R}_{o}=\int_{A_{e}} \mathbf{S}_{o}^{T} \mathbf{C P} d A \\
\Pi_{o}=\frac{1}{2} \int_{A_{e}} \mathbf{S}_{o}^{T} \mathbf{C S}_{o} d A+\int_{A_{e}} \mathbf{S}_{t}^{T} \mathbf{C} \mathbf{S}_{t} d A \\
+\int_{A_{e}} \mathbf{S}_{t}^{T} \mathbf{C S}_{o} d A
\end{gathered}
$$

The unknown vector, $\lambda$, contains the Lagrange multipliers used for enforcing the constraint equations, Eq. (30). In matrix form, the hybrid energy functional, $\Pi_{H}$, can be rewritten as

$$
\begin{aligned}
\Pi_{H} & =\frac{1}{2} \hat{\mathbf{b}}^{T} \hat{\mathbf{H}} \hat{\mathbf{b}}+{ }^{b} \hat{\mathbf{R}}_{o}^{T} \hat{\mathbf{b}}-{ }^{v} \mathbf{R}_{o}^{T} \mathbf{v} \\
& +{ }^{b} \hat{\mathbf{R}}_{t}^{T} \hat{\mathbf{b}}+\hat{\mathbf{b}}^{T} \hat{\mathbf{G}} \mathbf{v}+\Pi_{o}
\end{aligned}
$$

where

$$
\begin{gathered}
\hat{\mathbf{b}}^{T}=\left\{\mathbf{b}^{T}, \boldsymbol{\lambda}^{T}\right\}, \hat{\mathbf{H}}=\left[\begin{array}{cc}
\mathbf{H} & \mathbf{c}^{T} \\
\mathbf{c} & \mathbf{0}
\end{array}\right] \\
{ }^{b} \hat{\mathbf{R}}_{o}^{T}=\left\{{ }^{b} \mathbf{R}_{o}^{T}, \mathbf{0}^{T}\right\},{ }^{b} \hat{\mathbf{R}}_{t}^{T}=\left\{{ }^{b} \mathbf{R}_{t}^{T}, \mathbf{0}^{T}\right\} \\
\hat{\mathbf{G}}=\left[\begin{array}{c}
\mathbf{G} \\
\mathbf{0}
\end{array}\right]
\end{gathered}
$$

In accordance with the concept of energy minimization, the first variation of the hybrid energy functional with respect to the unknown vector $\hat{\mathbf{b}}$ of generalized coordinates yields

$$
\delta \hat{\mathbf{b}}^{T}\left(\hat{\mathbf{H}} \hat{\mathbf{b}}+{ }^{b} \hat{\mathbf{R}}_{o}+{ }^{b} \hat{\mathbf{R}}_{t}-\hat{\mathbf{G}} \mathbf{v}\right)=\mathbf{0}
$$

or

$$
\hat{\mathbf{b}}=\hat{\mathbf{H}}^{-1}\left(\hat{\mathbf{G}} \mathbf{v}-{ }^{b} \hat{\mathbf{R}}_{o}-{ }^{b} \hat{\mathbf{R}}_{t}\right)
$$

With this explicit solution form, the hybrid energy functional becomes

$$
\Pi_{H}=-\frac{1}{2} \mathbf{v}^{T} \mathbf{k} \mathbf{v}+\mathbf{f}_{o}^{T} \mathbf{v}+\mathbf{f}_{t}^{T} \mathbf{v}+\Pi_{o}
$$

in which the stiffness matrix $\mathbf{k}$ and the resultant force (load) vector $\mathbf{f}$ are defined as

$$
\mathbf{k}=\hat{\mathbf{G}}^{T} \hat{\mathbf{H}}^{-1} \hat{\mathbf{G}}, \mathbf{f}_{o}^{T}={ }^{b} \hat{\mathbf{R}}_{o}^{T} \hat{\mathbf{H}}^{-1} \hat{\mathbf{G}}-{ }^{\nu} \hat{\mathbf{R}}_{o}^{T}
$$

and

$$
\mathbf{f}_{t}^{T}={ }^{b} \hat{\mathbf{R}}_{t}^{T} \hat{\mathbf{H}}^{-1} \hat{\mathbf{G}}
$$

Associated with applied tractions, $q^{+}(x, y)$ and $q^{-}(x, y)$, on the upper and lower surfaces of the element, the load vector requires the explicit form of the particular solution, $\mathbf{S}_{o}$, to the equilibrium equations. This requirement is circumvented by computing the potential energy of external forces from

$$
\begin{aligned}
\mathbf{f}_{o}^{T} \mathbf{v} \int_{A} & {\left[q^{+}(x, y) u_{z}(x, y,+h / 2)\right.} \\
& \left.+q^{-}(x, y) u_{z}(x, y,-h / 2)\right] d A
\end{aligned}
$$

In order to evaluate the integral in Eq. (55), the weighted-average variables arising in the expression of the transverse displacement are approximated in terms of linear interpolation functions (only for the computation of the load vector), $N_{m}(x, y)$, as

$$
\left(w, w_{1}, w_{2}\right)=\sum_{m=1}^{3} N_{m}(x, y)\left(w_{(m)}^{(e)}, w_{1(m)}^{(e)}, w_{2(m)}^{(e)}\right)
$$

With this approximation, the load vector is recast as 


$$
\mathbf{f}_{o}^{T}=\left\{\mathbf{f}_{o 1}^{T}, \mathbf{f}_{o 2}^{T}, \mathbf{f}_{o 3}^{T}\right\}
$$

where $\mathbf{f}_{o k}^{T}=\left\{0,0, f_{w_{(k)}}, f_{w_{1(k)}} f_{w_{2}(k)}, 0,0,0,0,0,0,0,0\right\}$, with

$$
\begin{aligned}
f_{w_{(k)}} & =\int_{A_{e}} N_{k}\left[q^{+}(x, y)-q^{-}(x, y)\right] d A \\
f_{w_{1(k)}} & =\int_{A_{e}} N_{k}\left[q^{+}(x, y)+q^{-}(x, y)\right] d A \\
f_{w_{2(k)}} & =\frac{4}{5} \int_{A_{e}} N_{k}\left[q^{+}(x, y)-q^{-}(x, y)\right] d A
\end{aligned}
$$

Finally, the element equilibrium equation is obtained by requiring the first variation of the hybrid energy functional to vanish

$$
\delta \Pi_{H}=\delta \mathbf{v}^{T}\left(\mathbf{k} \mathbf{v}-\mathbf{f}_{o}-\mathbf{f}_{t}\right)=0
$$

For arbitrary variation of $\delta \mathbf{v}$, the element equilibrium equations become

$$
\mathbf{k} \mathbf{v}=\mathbf{f}_{o}+\mathbf{f}_{t}
$$

Assembly of the element stiffness matrices and force vectors leads to the structural equations of equilibrium as

$$
\mathbf{K V}=\mathbf{F}+\mathbf{F}_{c}
$$

in which $\mathbf{K}, \mathbf{V}$, and $\mathbf{F}$ represent the assembly of the element stiffness matrix and nodal and force vectors. The vector $\mathbf{F}_{c}$ arises from the presence of concentrated forces.

Although the transverse shear stresses can readily be obtained from the stress-strain relations given in Eq. (6), the presence of discrete material properties in the thickness direction causes discontinuous variation of transverse shear stresses. However, the true variation of the transverse shear stresses must be continuous through the thickness of the sandwich panel. The accurate computation of in-plane stresses and their first derivatives suggests that continuous transverse shear stresses, $\sigma_{x z}$ and $\sigma_{y z}$, can be obtained by integrating the in-plane equilibrium equations in the thickness direction. Similarly, the transverse normal stress component, $\sigma_{z z}$, is computed by integrating the transverse equilibrium equation in the thickness direction. Sandwich panels exhibit nonlinear deformation, with a discontinuous slope in the thickness direction due the severe changes in mechanical and thermal properties. However, in single-layer theories, through-the-thickness variation of the displacement components is approximated by polynomials. Such kinematic representation does not necessarily capture the accurate behavior of displacement components in the thickness direction because of the presence of discontinuous slopes at the interface of the core and face sheet. This inaccuracy is avoided by computing the displacement components from the integration of the strain components through the thickness. Details of the integration procedure for the computation of transverse shear, normal stress, and displacement components in the thickness direction are given in Appendix D.

\section{Numerical Results}

In order to demonstrate the capability of the present element, an unsymmetric laminate and a sandwich panel previously considered by Tessler et al. ${ }^{11}$ are studied. The validity of the present analysis results is established by comparison against the three-dimensional exact solutions presented by Tessler et al. The exact solutions were obtained using the PAND3D analysis program developed by Burton and Noor. ${ }^{16}$

The square geometry of the unsymmetric laminate and sandwich panel are described by $2 a=2 b=20 h$, with $h=0.5 \mathrm{in}$. The laminate consists of carbon/epoxy plies of equal thickness and a stacking sequence of $\left[0_{4} / 90_{4} / 0_{4} / 90_{4}\right]$. In the case of the sandwich panel, the face sheets at the bottom and top of the core are of equal thickness, $h_{f}=0.2 h$; hence, the core thickness, $h_{c}$, is $80 \%$ of the total panel thickness. The face sheet material is composed of carbon/epoxy plies, with stacking sequence $\left[0_{4} / 90_{2} / 0_{4} / 90_{2} / 0_{4}\right]$. Each carbon/ epoxy ply has Young's moduli of $E_{11}=22.9 \times 10^{6} \mathrm{psi}$ and $E_{22}=E_{33}=1.39 \times 10^{6} \mathrm{psi}$, shear moduli of $G_{12}=$ $G_{13}=0.864 \times 10^{6} \mathrm{psi}$ and $G_{23}=0.368 \times 10^{6} \mathrm{psi}$, Poisson's ratios of $v_{12}=v_{13}=0.32$ and $v_{23}=0.48$, and thermal expansion coefficients of $\alpha_{1}=0.01 \times 10^{-6} /{ }^{\circ} \mathrm{F}$ and $\alpha_{2}=\alpha_{3}=11.5 \times 10^{-6} /{ }^{\circ} \mathrm{F}$. The core is made of soft PVC (foam) with isotropic material properties specified as $E_{c}=15 . \times 10^{3} \mathrm{psi}, v_{c}=0.3$, and $\alpha_{c}=15.64 \times 10^{-6} /$ ${ }^{\circ} \mathrm{F}$. The shear correction factors that appear in matrix $\mathbf{G}$ of Eq. (B9) are $c_{y z}=c_{x z}=0.8939$ for the unsymmetric laminate and $c_{y z}=0.6161$ and $c_{x z}=0.5536$ for the sandwich panel.

In accordance with the $\{3,2\}$-order plate theory, the boundary conditions are enforced as

$$
\begin{aligned}
& u=w=w_{1}=w_{2}=w_{, x}=w_{1, x}=w_{2, x}=\theta_{y}=0 \\
& \text { for } y=0 \text { and } y=2 b \text {, and } \\
& v=w=w_{1}=w_{2}=w_{, y}=w_{1, y}=w_{2, y}=\theta_{x}=0
\end{aligned}
$$

As shown in Fig. 3, the non-uniform temperature distributions on the top and bottom surface are, respectively, specified by $T^{+}(x, y)=300 T(x, y)^{\circ} \mathrm{F}$ and $T^{-}(x, y)=60 T(x, y)^{\circ} \mathrm{F}$, where $T(x, y)=\sin (\pi x / 2 a)$ $x \cos (\pi y / 2 b)$. A total of 2500 elements are employed in the finite element discretization, leading to 16250 degrees of freedom. 


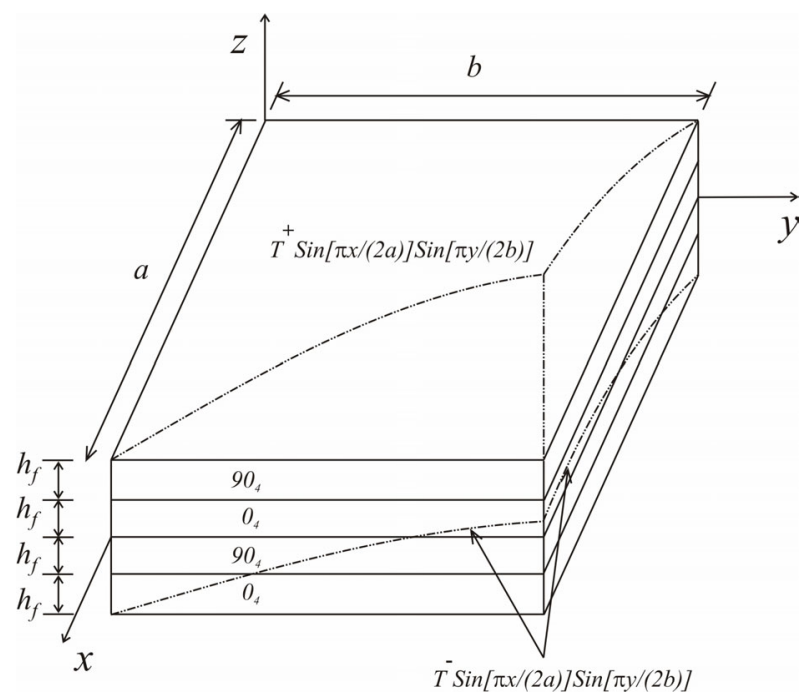

(a)

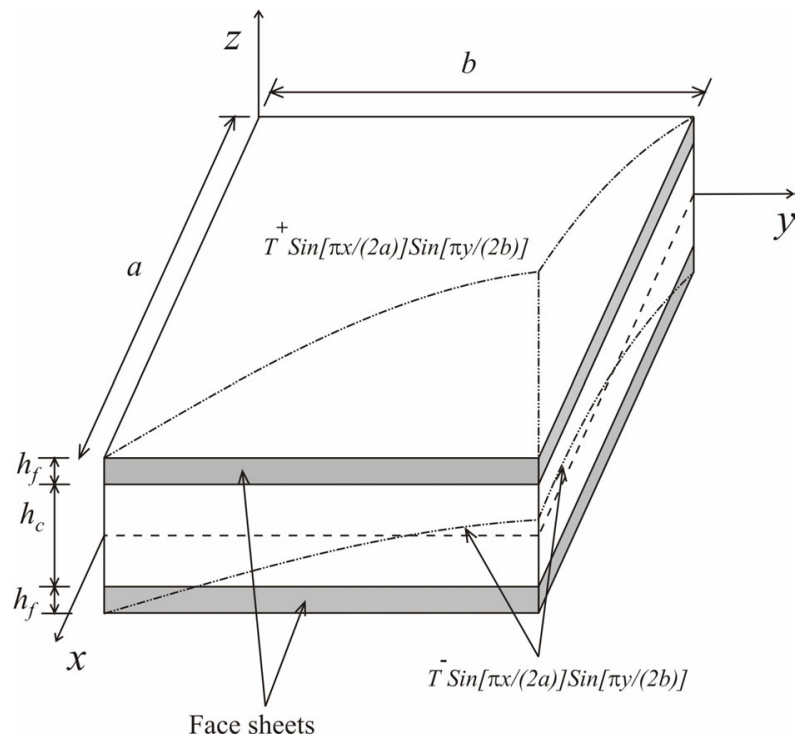

(b)

Fig. 3 Non-uniform surface temperature applied to (a) the $\left[0_{4} / 90_{2} / 0_{4} / 90_{2} / 0_{4}\right]$ laminate and (b) the sandwich panel.

The deformed configurations of the laminate and the sandwich panel are shown in Fig. 4. The laminate experiences significant bending and the upper surface bulges out more than the lower surface. In the case of the sandwich panel, there is no significant bending of the lower face sheet; however, the upper face sheet exhibits significant bending or it bulges out. The unsymmetric stacking sequence of the laminate enables the coupling between the in-plane stretching and transverse bending due to the non-zero values of the components

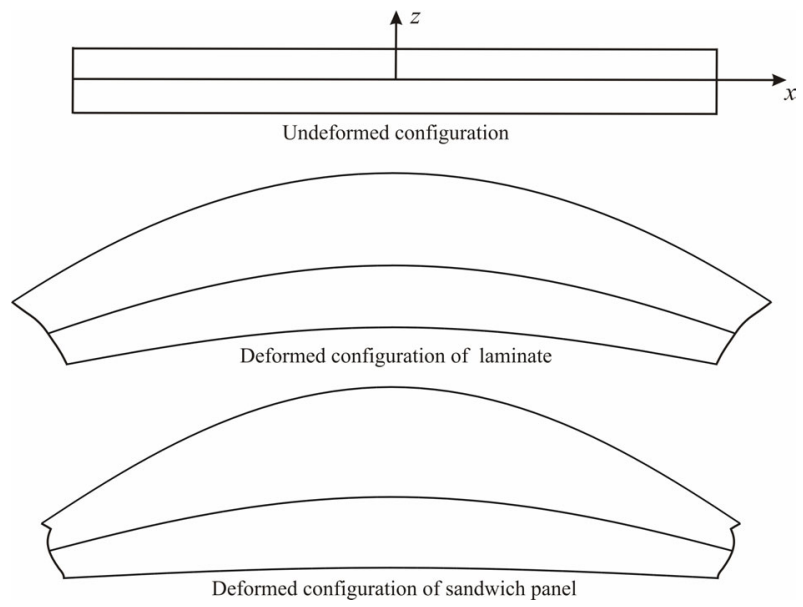

Fig. 4 Cross-sectional view of the deformed laminate and the sandwich panel subjected to thermal loading.

of matrix $\mathbf{B}$ of Eq. (B3). The variation of $u_{x}$ and $u_{z}$ as observed in Fig. 5 shows non-linear behavior, and the finite element solution is in close agreement with the exact solution. The nonlinear variation of the displacement components suggests the necessity of higher-order theories for thick laminates. Comparisons of the mechanical strains $\varepsilon_{x x}^{M}=\varepsilon_{x x}-\alpha_{x x} \Delta T$ and $\varepsilon_{z z}^{M}=\varepsilon_{z z}-$ $\alpha_{z z} \Delta T$ are shown in Fig. 6 . The magnitude of the inplane normal strain is comparable to that of the transverse normal strain due to the steep variation of the temperature in the thickness direction. The solutions from the present analysis for the in-plane normal stress $\sigma_{x x}$ and the transverse shear stress $\sigma_{x z}$ are in close agreement with the exact solution, as observed in Fig. 7. The process of integrating the equilibrium equation to obtain the transverse shear stress proves to be effective enough, and the solution satisfies the zero shear stress conditions at the top and bottom surfaces. Considering the complex, meandering nature of the transverse normal stress $\sigma_{z z}$ shown in Fig. 7c, the finite element results are in acceptable agreement. In the case of lower-order theories, such as the $\{1,2\}$ theory, the variation of $\sigma_{z z}$ becomes less accurate for laminates with low span-to-thickness ratios, as shown by Tessler et al. ${ }^{11}$

In the case of sandwich panels, the presence of a soft core in between stiff face sheets gives rise to a highly nonlinear behavior of the displacements. As observed from Fig. 8, apart from being nonlinear, the in-plane displacement $u_{x}$ has a slope discontinuity at the interface of the core and face sheets. The close proximity of the present solution to the exact solution is due to the utilization of the process of displacement recovery from integration of strain components. The variation of $u_{z}$ is 

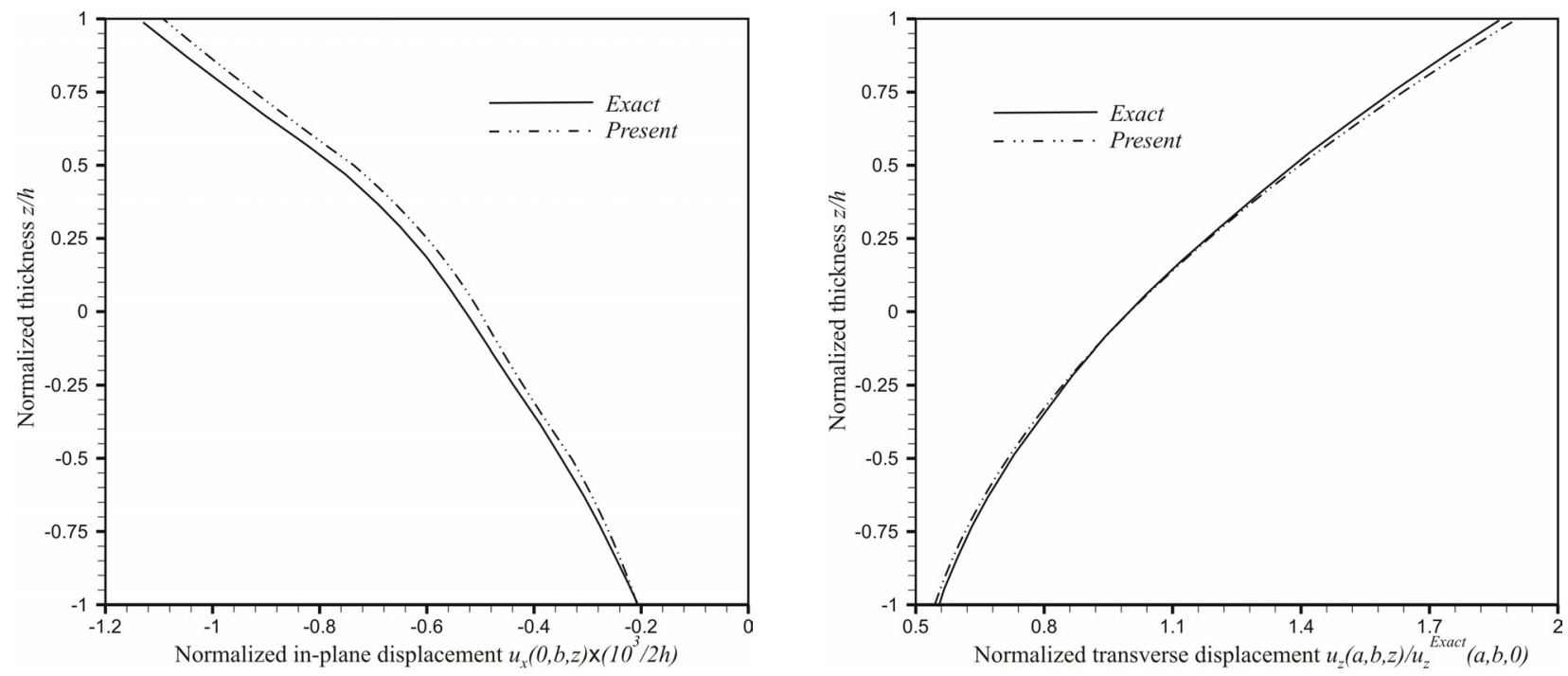

Fig.5 Through-the-thickness in-plane and transverse displacements, $u_{x}$ and $u_{z}$, for a $\left[0_{4} / 90_{2} / 0_{4} / 90_{2} / 0_{4}\right]$ laminate.
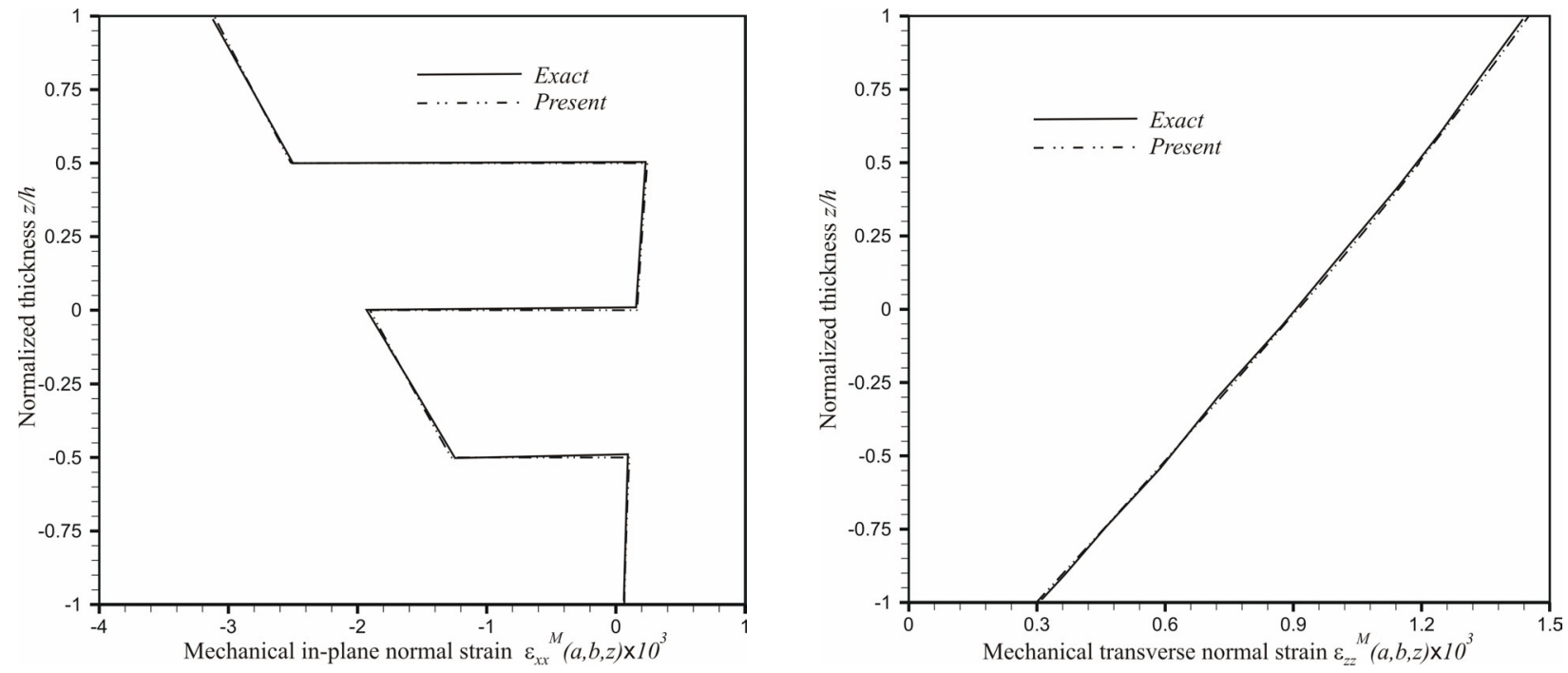

Fig. 6 Through-the-thickness mechanical in-plane normal and mechanical transverse normal strains, $\varepsilon_{x x}^{M}$ and $\varepsilon_{z z}^{M}$, for a $\left[0_{4} / 90_{2} / 0_{4} / 90_{2} / 0_{4}\right]$ laminate. 

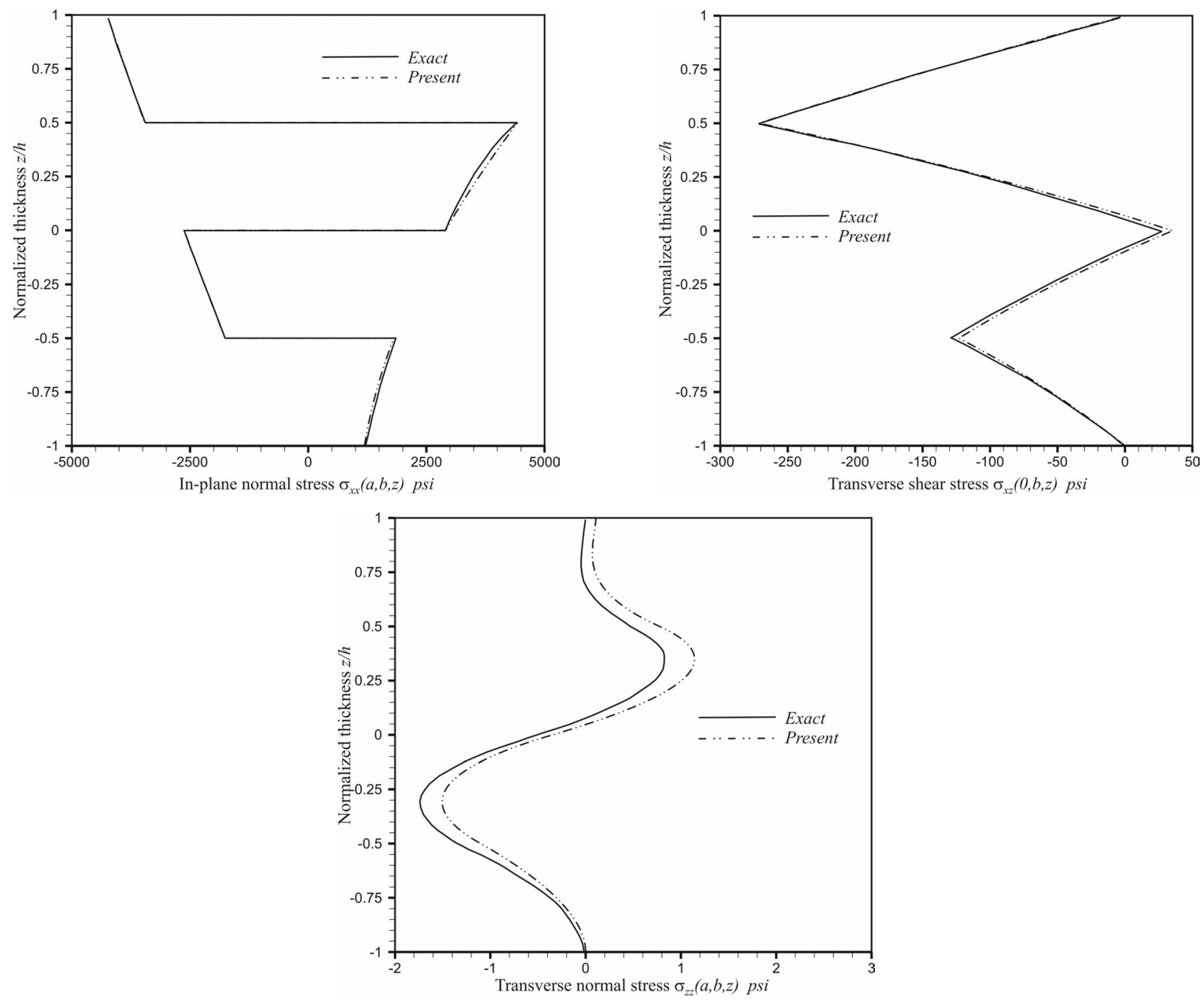

Fig. 7 Through-the-thickness in-plane normal, transverse shear, and transverse normal stresses, $\sigma_{x x}, \sigma_{x z}$, and $\sigma_{z z}$, for a $\left[0_{4} / 90_{2} / 0_{4} / 90_{2} / 0_{4}\right]$ laminate.
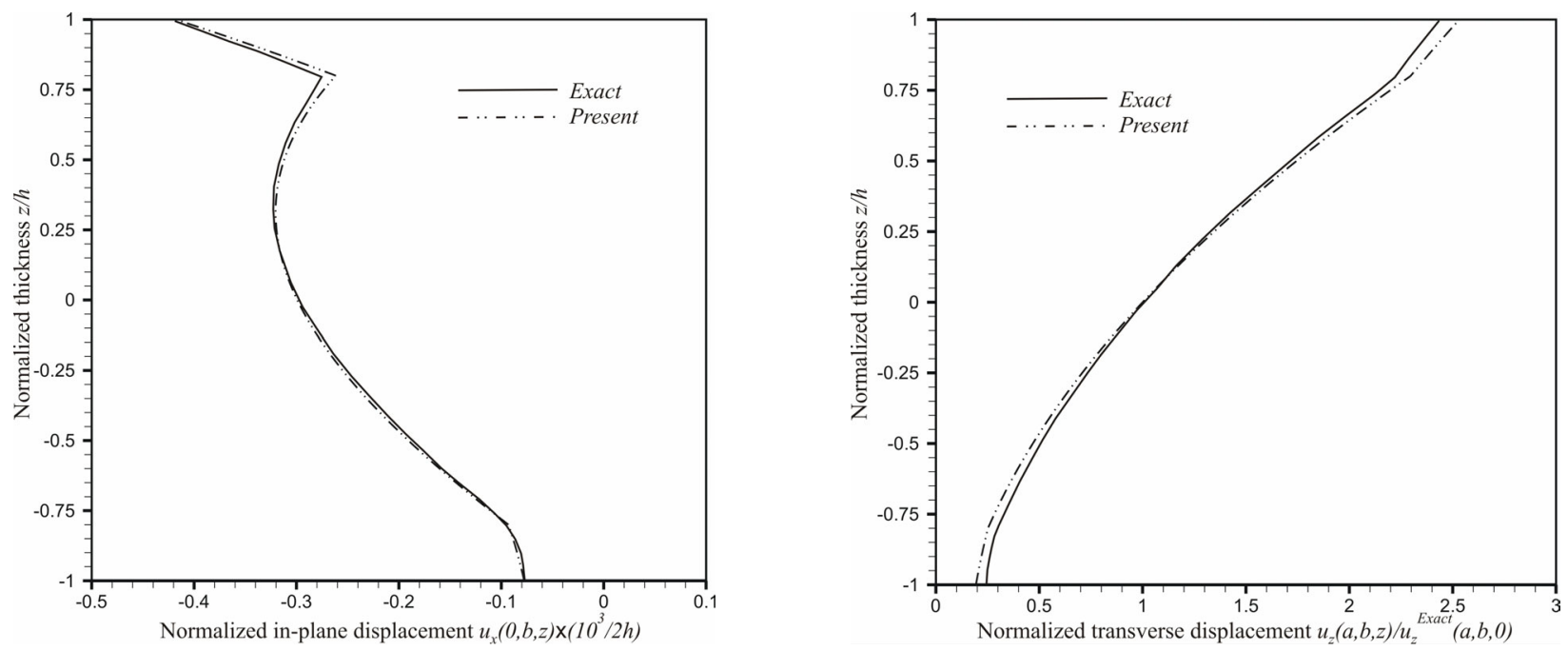

Fig. 8 Through-the-thickness in-plane and transverse displacements, $u_{x}$ and $u_{z}$, for a sandwich panel. 
fairly accurate, as illustrated in Fig. 8b. The results of the mechanical strains, $\varepsilon_{z z}^{M}$ and $\varepsilon_{z z}^{M}$, shown in Fig. 9, reveal that even in this case the transverse normal stain is comparable to the normal in-plane stain. As depicted in Fig. 10, $\sigma_{x x}$ and are in close agreement with the exact solution. The transverse shear stress follows a zig-zag path in the face sheets and the finite element solution is able to capture this pattern very accurately, apart from satisfying the zero shear stress criteria on the top and bottom surfaces. The transverse normal stress $\sigma_{z z}$ has a parabolic nature and, even though the initial assumption of $\sigma_{z z}$ in Eq. (11) is devoid of any quadratic terms, the equilibrium equation integration process recovers the correct solution although the magnitude of $\sigma_{z z}$ in the core area is slightly underestimated in the present analysis.

\section{Conclusions}

A new sandwich element based on the $\{3,2\}$-order plate theory in conjunction with the hybrid energy functional has been developed and validated for thermoelastic analysis of composite structures with sandwich constructions. The validation was established by comparison against the exact solution for a simply supported, unsymmetric, graphite/epoxy laminate and a sandwich panel made of a soft PVC core with graphite/ epoxy face sheets subjected to a non-uniform temperature field. This new computationally efficient element captures the correct variation of deformations in the thickness direction, as they are evaluated from a posteriori calculations of equilibrium equations.

\section{References}

${ }^{1}$ Das, M., Barut, A., Madenci, E., and Ambur, D. R., "A Triangular Sandwich Element for Complete Stress Fields Based on a Single-Layer Theory," Sixth International Conference on Sandwich Structures, edited by J. R. Vinson, Y. D. S. Rajapakse, and L. A. Carlsson, CRC Press, Boca Raton, FL, pp. 879-894.

${ }^{2}$ Barut, A., Madenci, W., and Tessler, A., "Nonuniform Thermoelastic Analysis of Composite Panels Under Non-Uniform Temperature Distribution," International Journal of Solids and Structures, Vol. 37, 2000, pp. 3681-3713.

${ }^{3}$ Chen, W. J., Lin, P. D., and Chen, L. W., "Thermal Buckling Behavior of Thick Composite Laminated Plates Under Nonuniform Temperature Distribution," Computers and Structures, Vol. 41, 1991, pp. 637-645.

${ }^{4}$ Ganapathi, M., and Touratier, M., "A Study on Thermal Postbuckling Behavior of Laminated Composite Plates Using a Shear Flexible Finite Element," Finite Elements in Analysis and Design, Vol. 28, 1997, pp. 115-135.

${ }^{5}$ Lee, D. M., and Lee, I., "Vibration Behavior of Thermally Postbuckled Anisotropic Plates Using FirstOrder Shear Deformable Plate Theory," Computers and Structures, Vol. 63, 1997, pp. 371-387.
${ }^{6}$ Rolfes, R., Noor, A. K., and Sparr, H., "Evaluation of Transverse Thermal Stress in Composite Plates Based on First-Order Shear Deformation Theory," Computer Methods in Applied Mechanical Engineering, Vol. 167, 1998, pp. 355-368.

${ }^{7}$ Park, J. W., and Kim, Y. H., "Re-Analysis Procedure for Laminated Plates Using FSDT Finite Element Models," Computational Mechanics, Vol. 29, 2002, pp. 226-242.

${ }^{8}$ Thankam, V. S., Singh, G. S., Rao, G. V., and Rath, A. K., "Thermal Postbuckling Behavior of Laminated Plates Using Shear-Flexible Elements Based on Coupled-Displacement Field," Composite Structures, Vol. 59, 2003, pp. 351-359.

${ }^{9}$ Kant, T., and Khare, R. K., "Finite Element Thermal Stress Analysis of Composite Laminates Using a Higher-Order Theory," Journal of Thermal Stresses, Vol. 17, 1994, pp. 229-255.

${ }^{10}$ Naganarayana, B. P., Rama Mohan, P., and Pratap, G., "Accurate Thermal Stress Prediction Using $C^{0}-$ Continuous Higher-Order Shear Deformable Elements," Computer Methods in Applied Mechanical Engineering, Vol. 144, 1997, pp. 61-75.

${ }^{11}$ Tessler, A., Annett, M. S., and Gendron, G., "A $\{1,2\}$-Order Plate Theory Accounting for Three-Dimensional Thermoelastic Deformation in Thick Composites and Sandwich Laminates," Computers and Structures, Vol. 52, 2000, pp. 67-84.

${ }^{12}$ Ali, J. S. M., Bhaskar, K., and Varadna, T. K., "A New Theory for Accurate Thermal/Mechanical Flexure Analysis of Symmetric Laminated Plates," Composite Structures, Vol. 45, 1999, pp. 227-232

${ }^{13}$ Kapuria, A., Dumir, P. C., and Ahmed, A., "An Efficient Higher Order Zigzag Theory for Composite and Sandwich Beams Subjected to Thermal Loading," International Journal of Solids and Structures, Vol. 40, 2003, pp. 6613-6631.

${ }^{14}$ Xiaoping, S., and Liangxin, S., "Thermomechanical Buckling of Laminated Composite Plates with Higher-Order Transverse Shear Deformation," Computers and Structures, Vol. 53, 1994, pp. 1-7.

${ }^{15}$ Tessler, A., "An Improved Plate Theory of $\{1,2\}$ Order for Thick Composite Laminates," International Journal of Solids and Structures, Vol. 7, 1993, pp. 1811000.

${ }^{16}$ Burton, W. S., and Noor, A. K., "Three-Dimensional Solution for Thermo-Mechanical Stress in Sandwich Panels and Shells," Journal of Engineering Mechanics, Vol. 120, 1994, 2044-2071.

${ }^{17}$ Barut, A., Madenci, E., Anderson, T., and Tessler, A., "Equivalent Single-Layer Theory for a Complete Stress Field In Sandwich Panels Under Arbitrarily Distributed Loading," Composites Structures, Vol. 58, 2002, pp. 483-495.

${ }^{18}$ Anderson, T., Madenci, E., Fish, J., and Burton, S. W., "Analytical Solution of Finite-Geometry Composite Panels Under Transient Surface Loading," International Journal of Solids and Structures, Vol. 35, 1998, pp. 1219-1239. 


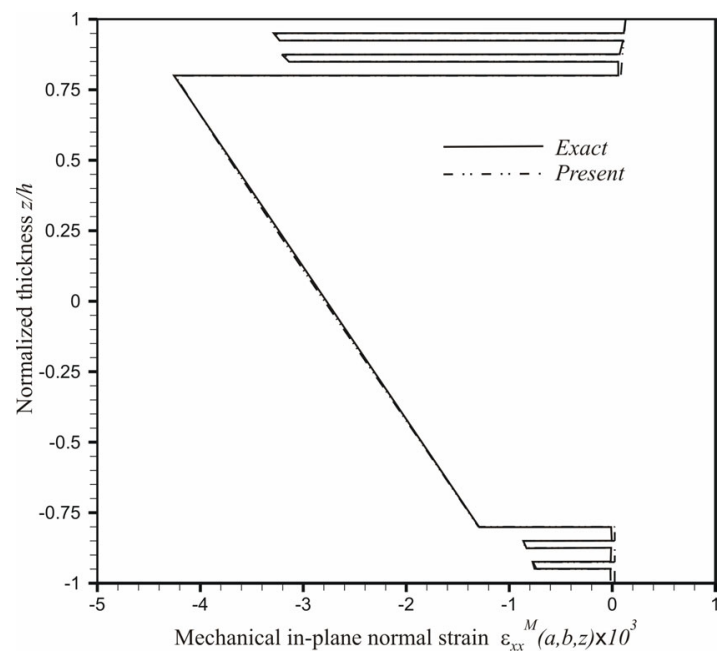

Fig.

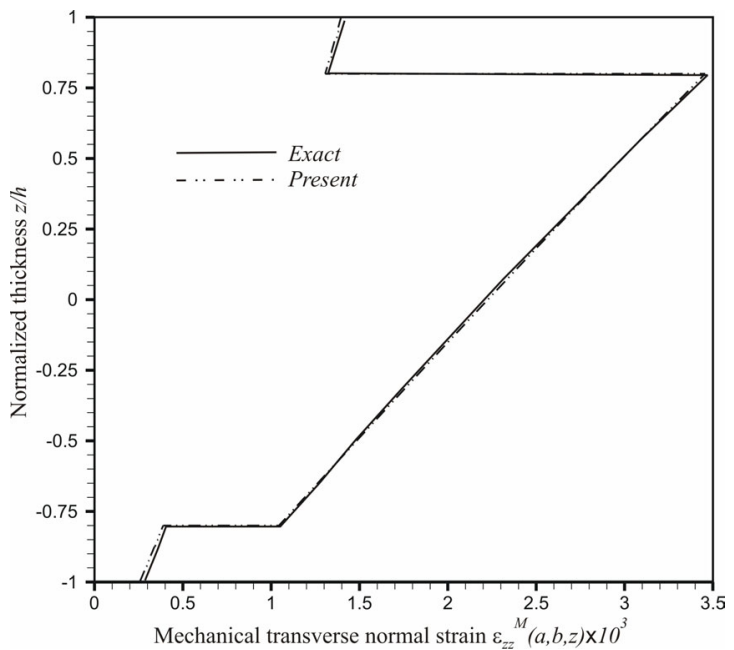

9 Through-the-thickness mechanical in-plane normal and mechanical transverse normal strains, $\varepsilon_{x x}^{M}$ and $\varepsilon_{z z}^{M}$, for a sandwich panel.
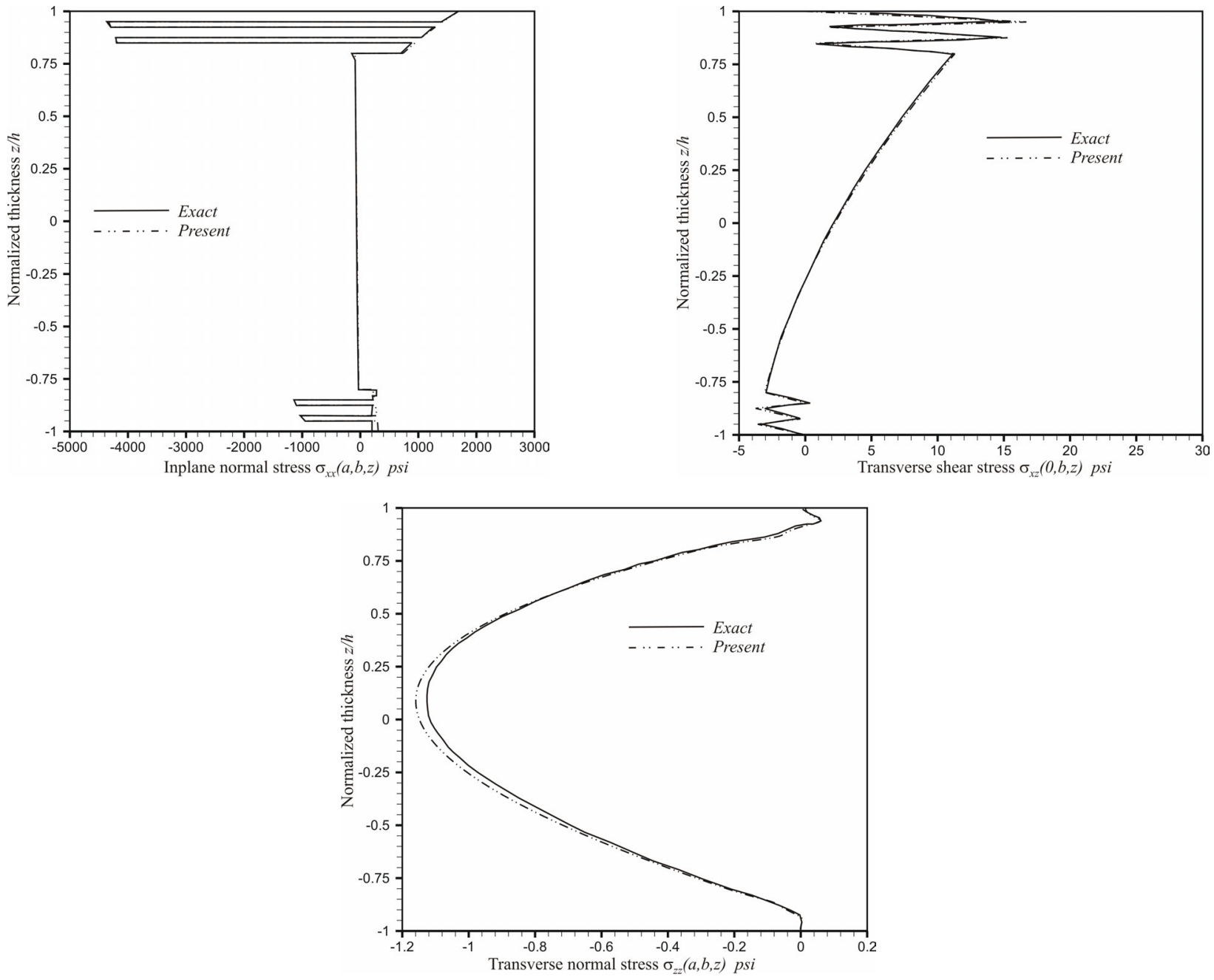

Fig. 10 Through-the-thickness in-plane normal, transverse shear, and transverse normal stresses, $\sigma_{x x}, \sigma_{x z}$ and $\sigma_{z z}$, for a sandwich panel. 


\section{Appendix A}

The vectors $\mathbf{q}_{\varepsilon}^{\prime}, \mathbf{q}_{\varepsilon}^{\prime \prime}, \mathbf{q}_{\kappa}^{\prime}$, and $\mathbf{q}_{\kappa}^{\prime \prime}$ in Eq. (11) are obtained from the matrix operation

$$
\left[\begin{array}{ll}
\mathbf{q}_{\varepsilon}^{\prime} & \mathbf{q}_{\kappa}^{\prime} \\
\mathbf{q}_{\varepsilon}^{\prime \prime} & \mathbf{q}_{\kappa}^{\prime \prime}
\end{array}\right]=\left[\begin{array}{ll}
S_{11} & S_{12} \\
S_{12} & S_{22}
\end{array}\right]^{-1}\left[\begin{array}{ll}
\mathbf{t}_{\varepsilon}^{\prime} & \mathbf{t}_{\kappa}^{\prime} \\
\mathbf{t}_{\delta}^{\prime \prime} & \mathbf{t}_{\kappa}^{\prime \prime}
\end{array}\right]
$$

where $S_{i j}(i, j=1,2), \mathbf{t}_{\alpha}^{\prime}$, and $\mathbf{t}_{\alpha}^{\prime \prime}(\alpha=\varepsilon, \kappa)$ are given as

$$
\begin{array}{cc}
{\left[S_{11}, S_{12}, S_{22}\right]=\sum_{k=1}^{K} \int_{h_{k-1}}^{h_{k}}\left[1, \phi, \phi^{2}\right] S_{33}^{(k)^{2}} d z_{k}} & \\
\mathbf{t}_{\alpha}^{\prime}=\left\{t_{k \alpha}^{\prime}\right\} \text { and } \mathbf{t}_{\alpha}^{\prime \prime}=\left\{t_{k \alpha}^{\prime \prime}\right\} & (k=1,7 ; \alpha=\varepsilon, \kappa)
\end{array}
$$

in which

$$
\left.\begin{array}{rl}
t_{j \varepsilon}^{\prime} & =\sum_{k=1}^{K} \int_{h_{k-1}}^{h_{k}} \varphi_{j \varepsilon} \hat{R}_{j 3}^{(k)} S_{33}^{(k)} d z_{k}, t_{j \kappa}^{\prime}=\sum_{k=1}^{K} \int_{h_{k-1}}^{h_{k}} \varphi_{j \kappa} \hat{R}_{j 3}^{(k)} S_{33}^{(k)} d z_{k} \\
t_{j \varepsilon}^{\prime \prime} & =\sum_{k=1}^{K} \int_{h_{k-1}}^{h_{k}} \varphi_{j \varepsilon} \phi \hat{R}_{j 3}^{(k)} S_{33}^{(k)} d z_{k}, \mathrm{t}_{\mathrm{j} \kappa}^{\prime \prime}=\sum_{k=1}^{K} \int_{h_{k-1}}^{h_{k}} \varphi_{j \kappa} \phi \hat{R}_{j 3}^{(k)} S_{33}^{(k)} d z_{k}
\end{array}\right\} \quad \quad(j=1,7 ; \text { no summation on } j)
$$

with

$$
\left\{\hat{R}_{j 3}^{(k)}\right\}=\left\{R_{13}^{(k)}, R_{23}^{(k)}, 1, R_{63}^{(k)}, R_{13}^{(k)}, R_{23}^{(k)}, R_{63}^{(k)}\right\}
$$

and

$$
\left\{\varphi_{j \varepsilon}\right\}=\left\{P_{0}, P_{0}, 1, P_{0}, P_{2}, P_{2}, P_{2}\right\}, \quad\left\{\varphi_{\mathrm{j} \kappa}\right\}=\left\{P_{1}, P_{1}, 1, P_{1}, P_{3}, P_{3}, P_{3}\right\}
$$

The computation of $r_{i}$ in Eq. (13) is done in the following manner:

where

$$
\left\{\begin{array}{l}
r_{1} \\
r_{2}
\end{array}\right\}=\left[\begin{array}{ll}
S_{11} & S_{12} \\
S_{12} & S_{22}
\end{array}\right]^{-1}\left\{\begin{array}{l}
l_{1} \\
l_{2}
\end{array}\right\}
$$

$$
\begin{aligned}
& l_{1}=\sum_{k=1}^{K} \int_{h_{k-1}}^{h_{k}}\left(R_{13}^{(k)} S_{33}^{(k)} \alpha_{x x}^{(k)}+R_{23}^{(k)} S_{33}^{(k)} \alpha_{y y}^{(k)}+S_{33}^{(k)} \alpha_{z z}^{(k)}+R_{63}^{(k)} S_{33}^{(k)} \alpha_{x y}^{(k)}\right)\left(\bar{T}_{1}+\bar{T}_{2} \zeta\right) d z_{k} \\
& l_{2}=\sum_{k=1}^{K} \int_{h_{k-1}}^{h_{k}}\left(R_{13}^{(k)} S_{33}^{(k)} \alpha_{x x}^{(k)}+R_{23}^{(k)} S_{33}^{(k)} \alpha_{y y}^{(k)}+S_{33}^{(k)} \alpha_{z z}^{(k)}+R_{63}^{(k)} S_{33}^{(k)} \alpha_{x y}^{(k)}\right)\left(\bar{T}_{1}+\bar{T}_{2} \zeta\right) \phi d z_{k}
\end{aligned}
$$

\section{$\underline{\text { Appendix B }}$}

The matrices $\mathbf{A}, \mathbf{D}$, and $\mathbf{B}$ in Eq. (20) are defined as

$$
\mathbf{A}=\left[\begin{array}{lllllll}
A_{11} & A_{12} & A_{13} & A_{14} & A_{15} & A_{16} & A_{17} \\
& A_{22} & A_{23} & A_{24} & A_{25} & A_{26} & A_{27} \\
& & A_{33} & A_{34} & A_{35} & A_{36} & A_{37} \\
& & & A_{44} & A_{45} & A_{46} & A_{47} \\
& & & & A_{55} & A_{56} & A_{57} \\
\text { sym. } & & & & & A_{66} & A_{67} \\
& & & & & & A_{77}
\end{array}\right]
$$

17 


$$
\begin{aligned}
& \mathbf{D}=\left[\begin{array}{ccccccc}
D_{11} & D_{12} & D_{13} & D_{14} & D_{15} & D_{16} & D_{17} \\
& D_{22} & D_{23} & D_{24} & D_{25} & D_{26} & D_{27} \\
& & D_{33} & D_{34} & D_{35} & D_{36} & D_{37} \\
& & & D_{44} & D_{45} & D_{46} & D_{47} \\
& & & & D_{55} & D_{56} & D_{57} \\
\text { sym. } & & & & & D_{66} & D_{67} \\
& & & & & & D_{77}
\end{array}\right] \\
& \mathbf{B}=\left[\begin{array}{lllllll}
B_{11} & B_{12} & B_{13} & B_{14} & B_{15} & B_{16} & B_{17} \\
B_{21} & B_{22} & B_{23} & B_{24} & B_{25} & B_{26} & B_{27} \\
B_{31} & B_{32} & B_{33} & B_{34} & B_{35} & B_{36} & B_{37} \\
B_{41} & B_{42} & B_{43} & B_{44} & B_{45} & B_{46} & B_{47} \\
B_{51} & B_{52} & B_{53} & B_{54} & B_{55} & B_{56} & B_{57} \\
B_{61} & B_{62} & B_{63} & B_{64} & B_{65} & B_{66} & B_{67} \\
B_{71} & B_{72} & B_{73} & B_{74} & B_{75} & B_{76} & B_{77}
\end{array}\right]
\end{aligned}
$$

and the matrix $\mathbf{G}$ has the form

$$
\mathbf{G}=\left[\begin{array}{ll}
G_{11} & G_{12} \\
G_{12} & G_{22}
\end{array}\right]
$$

By integrating the strain energy density of the sandwich panel [i.e., the first integrand in Eq. (16)], through the thickness after substituting from Eqs. (4), (6), (10), and (11) in conjunction with Eq. (13), the components of matrices $\mathbf{A}, \mathbf{B}, \mathbf{D}$, and $\mathbf{G}$ are obtained as

$$
\begin{aligned}
& A_{i j}=\sum_{k=1}^{K} \int_{h_{k-1}}^{h_{k}}\left\{\hat{C}_{i j}^{(k)} \varphi_{i \varepsilon} \varphi_{j \varepsilon}+s_{33}^{(k)} \psi_{i \varepsilon} \psi_{j \varepsilon}\right\} d z_{k} \\
& \left.B_{i j}=\sum_{k=1}^{K} \int_{h_{k-1}}^{h_{k}}\left\{\hat{C}_{i j}^{(k)} \varphi_{i \varepsilon} \varphi_{j \kappa}+s_{33}^{(k)} \psi_{i \varepsilon} \psi_{j \kappa}\right\} d z_{k}\right\} \quad(i, j=1,7 ; \text { no summation on } i \text { and } j) \\
& D_{i j}=\sum_{k=1}^{K} \int_{h_{k-1}}^{h_{k}}\left\{\hat{C}_{i j}^{(k)} \varphi_{i \kappa} \varphi_{j \kappa}+s_{33}^{(k)} \psi_{i \kappa} \psi_{j \kappa}\right\} d z_{k}
\end{aligned}
$$

and

$$
G_{i j}=\sum_{k=1}^{K} \int_{h_{k-1}}^{h_{k}}\left\{\left[\frac{5}{4}\left(1-\xi^{2}\right)\right]^{2} \hat{C}_{i+3, j+3}\right\} d z_{k}
$$

in which $\hat{C}_{i j}^{(k)}$ is defined in matrix form as

$$
\left[\hat{C}_{i j}^{(k)}\right]=\left[\begin{array}{ccccccc}
\bar{C}_{11} & \bar{C}_{12} & 0 & \bar{C}_{16} & \bar{C}_{11} & \bar{C}_{12} & \bar{C}_{16} \\
& \bar{C}_{22} & 0 & \bar{C}_{26} & \bar{C}_{12} & \bar{C}_{22} & \bar{C}_{26} \\
& & 0 & 0 & 0 & 0 & 0 \\
& & & \bar{C}_{66} & \bar{C}_{16} & \bar{C}_{26} & \bar{C}_{66} \\
\text { sym. } & & & & \bar{C}_{11} & \bar{C}_{12} & \bar{C}_{16} \\
& & & & & \bar{C}_{22} & \bar{C}_{26} \\
& & & & & & \bar{C}_{66}
\end{array}\right]^{(k)}
$$

18

American Institute of Aeronautics and Astronautics 
and the functions $\varphi_{j \varepsilon}$ and $\varphi_{j \kappa}(j=1,7)$ are defined in the form

$$
\left.\begin{array}{l}
\psi_{j \varepsilon}=q_{j \varepsilon}^{\prime}+\phi q_{j \varepsilon}^{\prime \prime} \\
\psi_{j \kappa}=q_{j \kappa}^{\prime}+\phi q_{j \kappa}^{\prime \prime}
\end{array}\right\}
$$

$$
(j=1,7)
$$

Note that matrices $\mathbf{A}, \mathbf{D}$, and $\mathbf{G}$ are symmetric, whereas matrix $\mathbf{B}$ becomes nonsymmetric due to the presence of the $\psi_{j}$ 's.

Although the single-layer theory predicts the correct through-the-thickness variation of transverse shear strains for an isotropic panel, it does not consistently predict accurate results for composite sandwich panels having significant differences in material properties between the core and the face sheet. The commonly accepted approach referred to as the energy (transverse shear strain energy) matching technique is invoked in order to correct for the transverse shear strain behavior. This correction is invoked in matrix $\mathbf{G}$ as

$$
\mathbf{G}=\left[\begin{array}{cc}
c_{y z}^{2} G_{11} & c_{y z} c_{x z} G_{12} \\
c_{y z} c_{x z} G_{12} & c_{x z}^{2} G_{22}
\end{array}\right]
$$

where $c_{y z}$ and $c_{x z}$ are known as the shear correction factors. The shear correction factors improve the global transverse displacements of the sandwich panel. The exact transverse shear strain energies are related through the shear correction factors as

$$
U_{\gamma_{\alpha z}}^{(\text {exact })}=\frac{1}{c_{\alpha z}^{2}} U_{\gamma_{\alpha z}}^{\text {(uncorrected })} \quad(\alpha=x, y)
$$

in which $c_{\alpha z}$, with $(\alpha=x, y)$, represents the transverse shear correction factors.

Determination of the transverse shear correction factors for a particular material system requires the exact solution of benchmark problems for which the transverse shear strain energy must be independent of the geometry and loading. This leads to the shear correction factor being dependent on a particular material system only.

A square, simply supported, thin panel with a length-to-thickness ratio of 10000 under a double sinusoidal distributed loading is considered as a benchmark problem. Based on the analytical solution of the present singlelayer theory provided by Barut et al. ${ }^{17}$ and the exact solution developed by Anderson et al., ${ }^{18}$ the transverse shear strain energies $U_{\gamma_{\alpha z}}^{(\text {uncorrected })}$ and $U_{\gamma_{\alpha z}}^{(\text {exact })}$ of the panel can be expressed in the form

$$
\begin{aligned}
U_{\gamma_{\alpha z}}^{(\text {uncorrected })} & =\widehat{U}_{\gamma_{\alpha z}}^{\text {(uncorrected })} \int_{0}^{2 b} \int_{0}^{2 a} \operatorname{Sin}^{2}\left(\frac{\pi x}{2 a}\right) \operatorname{Sin}^{2}\left(\frac{\pi y}{2 b}\right) d x d y \\
U_{\gamma_{\alpha z}}^{(\text {exact })} & =\bar{U}_{\gamma_{\alpha z}}^{\text {(exact) }} \int_{0}^{2 b} \int_{0}^{2 a} \operatorname{Sin}^{2}\left(\frac{\pi x}{2 a}\right) \operatorname{Sin}^{2}\left(\frac{\pi y}{2 b}\right) d x d y
\end{aligned}
$$

where $2 a$ and $2 b$ are the lengths of the panel along the $x$ - and $y$-axes and $\widehat{U}_{\gamma_{\alpha z}}^{(\text {uncorrected })}$ and $\widehat{U}_{\gamma_{\alpha z}}^{(\text {exact })}$ are given by

$$
\begin{gathered}
\widehat{U}_{\gamma_{\alpha z}}^{\text {(uncorrected })}=\sum_{k=1}^{N} \int_{z_{k}}^{z_{k+1}} G_{\alpha}\left(\gamma_{\alpha z 0}^{\text {(uncorrected })}(z)\right)^{2} d z \\
\widehat{U}_{\gamma_{\alpha z}}^{(\text {exact })}=\sum_{k=1}^{N} \int_{z_{k}}^{z_{k+1}} \frac{\left(\hat{\sigma}_{\alpha z}^{(\text {exact })}(z)\right)^{2}}{G_{\alpha}} d z
\end{gathered}
$$

where $G_{x}=G_{11}$ and $G_{y}=G_{22}$. Therefore, substituting from Eqs. (B11) and (B12) into Eq. (B10) results in the transverse shear correction factors, $c_{\alpha z}^{2}$, being dependent only on the material system in a simplified form

$$
c_{\alpha z}^{2}=\frac{\widehat{U}_{\gamma_{\alpha z}}^{\text {(uncorrected) }}}{\widehat{U}_{\gamma_{\alpha z}}^{(\text {exact })}}
$$$$
(\alpha=x, y)
$$ 
The components of the resultant thermal force vector $\mathbf{N}^{*}$ in Eq. (20) are defined as

$$
\begin{aligned}
& { }^{(0)} N_{x x}^{*}=-\sum_{k=1}^{K} \int_{h_{k-1}}^{h_{k}}\left[\left(q_{1 \varepsilon}^{\prime}+\phi q_{1 \kappa}^{\prime}\right)\left(r_{1}+\phi r_{2}\right) S_{33}^{(k)}\right. \\
& \left.-\left(\alpha_{x x}^{(k)} C_{11}^{(k)}+\alpha_{y y}^{(k)} C_{12}^{(k)}+\alpha_{x y}^{(k)} C_{16}^{(k)}\right)\left(\bar{T}_{1}+\bar{T}_{2} \zeta\right)\right] d z_{k} \\
& { }^{(0)} N_{y y}^{*}=-\sum_{k=1}^{K} \int_{h_{k-1}}^{h_{k}}\left[\left(q_{2 \varepsilon}^{\prime}+\phi q_{2 \kappa}^{\prime}\right)\left(r_{1}+\phi r_{2}\right) S_{33}^{(k)}\right. \\
& \left.-\left(\alpha_{x x}^{(k)} C_{12}^{(k)}+\alpha_{y y}^{(k)} C_{22}^{(k)}+\alpha_{x y}^{(k)} C_{26}^{(k)}\right)\left(\bar{T}_{1}+\bar{T}_{2} \zeta\right)\right] d z_{k} \\
& { }^{(0)} N_{z z}^{*}=-\sum_{k=1}^{K} \int_{h_{k-1}}^{h_{k}}\left[\left(q_{3 \varepsilon}^{\prime}+\phi q_{3 \kappa}^{\prime}\right)\left(r_{1}+\phi r_{2}\right) S_{33}^{(k)}\right] d z_{k} \\
& { }^{(0)} N_{x y}^{*}=-\sum_{k=1}^{K} \int_{h_{k-1}}^{h_{k}}\left[\left(q_{4 \varepsilon}^{\prime}+\phi q_{4 \kappa}^{\prime}\right)\left(r_{1}+\phi r_{2}\right) S_{33}^{(k)}\right. \\
& \left.-\left(\alpha_{x x}^{(k)} C_{16}^{(k)}+\alpha_{y y}^{(k)} C_{26}^{(k)}+\alpha_{x y}^{(k)} C_{66}^{(k)}\right)\left(\bar{T}_{1}+\bar{T}_{2} \zeta\right)\right] d z_{k} \\
& { }^{(1)} N_{x x}^{*}=-\sum_{k=1}^{K} \int_{h_{k-1}}^{h_{k}}\left[\left(q_{5 \varepsilon}^{\prime}+\phi q_{5 \kappa}^{\prime}\right)\left(r_{1}+\phi r_{2}\right) S_{33}^{(k)}\right. \\
& \left.-\left(\alpha_{x x}^{(k)} C_{11}^{(k)}+\alpha_{y y}^{(k)} C_{12}^{(k)}+\alpha_{x y}^{(k)} C_{16}^{(k)}\right)\left(\bar{T}_{1}+\bar{T}_{2} \zeta\right)\left(\frac{1}{6}-\frac{\zeta^{2}}{2}\right)\right] d z_{k} \\
& { }^{(1)} N_{y y}^{*}=-\sum_{k=1}^{K} \int_{h_{k-1}}^{h_{k}}\left[\left(q_{6 \varepsilon}^{\prime}+\phi q_{6 \kappa}^{\prime}\right)\left(r_{1}+\phi r_{2}\right) S_{33}^{(k)}\right. \\
& \left.-\left(\alpha_{x x}^{(k)} C_{12}^{(k)}+\alpha_{y y}^{(k)} C_{22}^{(k)}+\alpha_{x y}^{(k)} C_{26}^{(k)}\right)\left(\bar{T}_{1}+\bar{T}_{2} \zeta\right)\left(\frac{1}{6}-\frac{\zeta^{2}}{2}\right)\right] d z_{k} \\
& { }^{(1)} N_{x y}^{*}=-\sum_{k=1}^{K} \int_{h_{k-1}}^{h_{k}}\left[\left(q_{7 \varepsilon}^{\prime}+\phi q_{7 \kappa}^{\prime}\right)\left(r_{1}+\phi r_{2}\right) S_{33}^{(k)}\right. \\
& \left.-\left(\alpha_{x x}^{(k)} C_{16}^{(k)}+\alpha_{y y}^{(k)} C_{26}^{(k)}+\alpha_{x y}^{(k)} C_{66}^{(k)}\right)\left(\bar{T}_{1}+\bar{T}_{2} \zeta\right)\left(\frac{1}{6}-\frac{\zeta^{2}}{2}\right)\right] d z_{k}
\end{aligned}
$$

The components of the resultant thermal moment vector $\mathbf{M}^{*}$ in Eq. (20) are defined as

$$
\begin{aligned}
{ }^{(0)} M_{x x}^{*}= & -\sum_{k=1}^{K} \int_{h_{k-1}}^{h_{k}}\left[\left(q_{1 \varepsilon}^{\prime \prime}+\phi q_{1 \kappa}^{\prime \prime}\right)\left(r_{1}+\phi r_{2}\right) S_{33}^{(k)}\right. \\
& \left.-h \zeta\left(\alpha_{x x}^{(k)} C_{11}^{(k)}+\alpha_{y y}^{(k)} C_{12}^{(k)}+\alpha_{x y}^{(k)} C_{16}^{(k)}\right)\left(\bar{T}_{1}+\bar{T}_{2} \zeta\right)\right] d z_{k} \\
{ }^{(0)} M_{y y}^{*}= & -\sum_{k=1}^{K} \int_{h_{k-1}}^{h_{k}}\left[\left(q_{2 \varepsilon}^{\prime \prime}+\phi q_{2 \kappa}^{\prime \prime}\right)\left(r_{1}+\phi r_{2}\right) S_{33}^{(k)}\right. \\
& \left.-h \zeta\left(\alpha_{x x}^{(k)} C_{12}^{(k)}+\alpha_{y y}^{(k)} C_{22}^{(k)}+\alpha_{x y}^{(k)} C_{26}^{(k)}\right)\left(\bar{T}_{1}+\bar{T}_{2} \zeta\right)\right] d z_{k}
\end{aligned}
$$




$$
\begin{gathered}
{ }^{(0)} M_{z z}^{*}=-\sum_{k=1}^{K} \int_{h_{k-1}}^{h_{k}}\left[\left(q_{3 \varepsilon}^{\prime \prime}+\phi q_{3 K}^{\prime \prime}\right)\left(r_{1}+\phi r_{2}\right) S_{33}^{(k)}\right] d z_{k} \\
{ }^{(0)} M_{x y}^{*}=-\sum_{k=1}^{K} \int_{h_{k-1}}^{h_{k}}\left[\left(q_{4 \varepsilon}^{\prime \prime}+\phi q_{4 \kappa}^{\prime \prime}\right)\left(r_{1}+\phi r_{2}\right) S_{33}^{(k)}\right. \\
\left.-h \zeta\left(\alpha_{x x}^{(k)} C_{16}^{(k)}+\alpha_{y y}^{(k)} C_{26}^{(k)}+\alpha_{x y}^{(k)} C_{66}^{(k)}\right)\left(\bar{T}_{1}+\bar{T}_{2} \zeta\right)\right] d z_{k} \\
{ }^{(1)} M_{x x}^{*}=-\sum_{k=1}^{K} \int_{h_{k-1}}^{h_{k}}\left[\left(q_{5 \varepsilon}^{\prime \prime}+\phi q_{5_{K}}^{\prime \prime}\right)\left(r_{1}+\phi r_{2}\right) S_{33}^{(k)}\right. \\
\left.-h\left(\alpha_{x x}^{(k)} C_{11}^{(k)}+\alpha_{y y}^{(k)} C_{12}^{(k)}+\alpha_{x y}^{(k)} C_{16}^{(k)}\right)\left(\bar{T}_{1}+\bar{T}_{2} \zeta\right)\left(\frac{\zeta}{5}-\frac{\zeta^{3}}{3}\right)\right] d z_{k} \\
{ }^{(1)} M_{y y}^{*}=-\sum_{k=1}^{K} \int_{h_{k-1}}^{h_{k}}\left[\left(q_{6 \varepsilon}^{\prime \prime}+\phi q_{6 \kappa}^{\prime \prime}\right)\left(r_{1}+\phi r_{2}\right) S_{33}^{(k)}\right. \\
\left.-h\left(\alpha_{x x}^{(k)} C_{12}^{(k)}+\alpha_{y y}^{(k)} C_{22}^{(k)}+\alpha_{x y}^{(k)} C_{26}^{(k)}\right)\left(\bar{T}_{1}+\bar{T}_{2} \zeta\right)\left(\frac{\zeta}{5}-\frac{\zeta^{3}}{3}\right)\right] d z_{k} \\
{ }^{(1)} M_{x y}^{*}=-\sum_{k=1}^{K} \int_{h_{k-1}}^{h_{k}}\left[\left(q_{7 \varepsilon}^{\prime \prime}+\phi q_{7 \kappa}^{\prime \prime}\right)\left(r_{1}+\phi r_{2}\right) S_{33}^{(k)}\right. \\
\left.-h\left(\alpha_{x x}^{(k)} C_{16}^{(k)}+\alpha_{y y}^{(k)} C_{26}^{(k)}+\alpha_{x y}^{(k)} C_{66}^{(k)}\right)\left(\bar{T}_{1}+\bar{T}_{2} \zeta\right)\left(\frac{\zeta}{5}-\frac{\zeta^{3}}{3}\right)\right] d z_{k}
\end{gathered}
$$

\section{Appendix C}

The matrices $\mathbf{b}$ and $\mathbf{P}$ in Eq. (33) can be expressed as

$$
\begin{aligned}
\mathbf{b}^{T}=\left\{\begin{array}{l}
{ }^{(0)} \boldsymbol{\alpha}_{x x}^{T},{ }^{(0)} \boldsymbol{\alpha}_{y y}^{T},{ }^{(0)} \boldsymbol{\alpha}_{z z}^{T},{ }^{(0)} \boldsymbol{\alpha}_{x y}^{T},{ }^{(1)} \boldsymbol{\alpha}_{x x}^{T},{ }^{(1)} \boldsymbol{\alpha}_{y y}^{T}, \\
\left.{ }^{\left({ }^{(1)}\right.} \boldsymbol{\alpha}_{x y}^{T},{ }^{(0)} \boldsymbol{\beta}_{x x}^{T},{ }^{(0)} \boldsymbol{\beta}_{y y}^{T},{ }^{(0)} \boldsymbol{\beta}_{z z}^{T},{ }^{(0)} \boldsymbol{\beta}_{x y}^{T},{ }^{(1)} \boldsymbol{\beta}_{x x}^{T},{ }^{(1)} \boldsymbol{\beta}_{y y}^{T},{ }^{(1)} \boldsymbol{\beta}_{x y}^{T}{ }^{(0)} \boldsymbol{\rho}_{y z}^{T},{ }^{(0)} \boldsymbol{\rho}_{x z}^{T}\right\}
\end{array}\right.
\end{aligned}
$$

where

$$
\begin{gathered}
{ }^{(\ell)} \boldsymbol{\alpha}_{\xi \eta}^{T}=\left\{{ }^{(\ell)} \boldsymbol{\alpha}_{\xi \eta(0)}^{T},{ }^{(\ell)} \boldsymbol{\alpha}_{\xi \eta(1)}^{T},{ }^{(\ell)} \boldsymbol{\alpha}_{\xi \eta(2)}^{T},{ }^{(\ell)} \boldsymbol{\alpha}_{\xi \eta(3)}^{T},{ }^{(\ell)} \boldsymbol{\alpha}_{\xi \eta(4)}^{T}\right\} \\
{ }^{(0)} \boldsymbol{\alpha}_{z z}^{T}=\left\{{ }^{(0)} \boldsymbol{\alpha}_{z z(0)}^{T},{ }^{(0)} \boldsymbol{\alpha}_{z z(1)}^{T},{ }^{(0)} \boldsymbol{\alpha}_{z z(2)}^{T}\right\} \\
{ }^{(\ell)} \boldsymbol{\beta}_{\xi \eta}^{T}=\left\{{ }^{(\ell)} \boldsymbol{\beta}_{\xi \eta(0)}^{T},{ }^{(\ell)} \boldsymbol{\beta}_{\xi \eta(1)}^{T},{ }^{(\ell)} \boldsymbol{\beta}_{\xi \eta(2)}^{T},{ }^{(\ell)} \boldsymbol{\beta}_{\xi \eta(3)}^{T},{ }^{(\ell)} \boldsymbol{\beta}_{\xi \eta(4)}^{T},\right\} \\
{ }^{(0)} \boldsymbol{\beta}_{z z}^{T}=\left\{{ }^{(0)} \boldsymbol{\beta}_{z z(0)}^{T},{ }^{(0)} \boldsymbol{\beta}_{z z(1)}^{T},{ }^{(0)} \boldsymbol{\beta}_{z z(2)}^{T}\right\} \\
{ }^{(0)} \boldsymbol{\beta}_{\xi z}^{T}=\left\{{ }^{(0)} \boldsymbol{\rho}_{\xi z(0)}^{T},{ }^{(0)} \boldsymbol{\beta}_{\xi z(1)}^{T},{ }^{(0)} \boldsymbol{\beta}_{\xi z(2)}^{T}\right\}
\end{gathered}
$$

and 


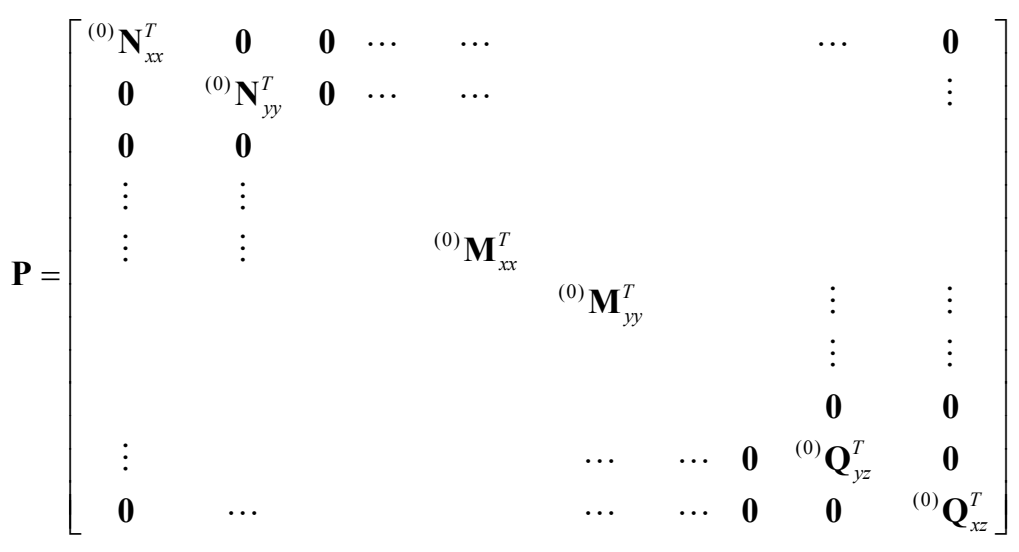

where

$$
\begin{aligned}
& { }^{(l)} \mathbf{N}_{\xi \eta}^{T}=\left\{{ }^{(l)} \mathbf{N}_{\xi \eta(0)}^{T},{ }^{(l)} \mathbf{N}_{\xi \eta(1)}^{T},{ }^{(l)} \mathbf{N}_{\xi \eta(2)}^{T},{ }^{(l)} \mathbf{N}_{\xi \eta(3)}^{T},{ }^{(l)} \mathbf{N}_{\xi \eta(4)}^{T}\right\} \\
& { }^{(0)} \mathbf{N}_{z z}^{T}=\left\{{ }^{\left({ }^{0}\right.} \mathbf{N}_{z z(0)}^{T},{ }^{(0)} \mathbf{N}_{z z(1)}^{T},{ }^{(0)} \mathbf{N}_{z z(2)}^{T}\right\} \\
& { }^{\left({ }^{(l)}\right.} \mathbf{M}_{\xi \eta}^{T}=\left\{{ }^{(l)} \mathbf{M}_{\xi \eta(0)}^{T},{ }^{(l)} \mathbf{M}_{\xi \eta(1)},{ }^{T}{ }^{(l)} \mathbf{M}_{\xi \eta(2)}^{T},{ }^{(l)} \mathbf{M}_{\xi \eta(3)}^{T},{ }^{(l)} \mathbf{M}_{\xi \eta(4)}^{T}\right\} \\
& { }^{(0)} \mathbf{M}_{z z}^{T}=\left\{{ }^{(0)} \mathbf{M}_{z z(0)}^{T},{ }^{(0)} \mathbf{M}_{z z(1)}^{T},{ }^{(0)} \mathbf{M}_{z z(2)}^{T}\right\} \\
& { }^{(0)} \mathbf{Q}_{\xi \eta}^{T}=\left\{{ }^{(0)} \mathbf{Q}_{\xi \eta(0)}^{T},{ }^{(0)} \mathbf{Q}_{\xi \eta(1)}^{T},{ }^{(0)} \mathbf{Q}_{\xi \eta(2)}^{T},{ }^{(0)} \mathbf{Q}_{\xi \eta(3)}^{T}\right\}
\end{aligned}
$$

The matrix $\mathbf{L}^{\prime(k)}$ in Eq. (37), which consists of the interpolation functions, is defined as

$$
\mathbf{L}^{\prime(k)}=\left[\begin{array}{cccccccccc}
N_{1}(s) & N_{2}(s) & 0 & 0 & 0 & 0 & 0 & 0 & 0 & 0 \\
0 & 0 & N_{1}(s) & N_{2}(s) & 0 & 0 & 0 & 0 & 0 & 0 \\
0 & 0 & 0 & 0 & H_{1}(s) & H_{2}(s) & 0 & 0 & 0 & 0 \\
0 & 0 & 0 & 0 & 0 & 0 & H_{1}(s) & H_{2}(s) & 0 & 0 \\
0 & 0 & 0 & 0 & 0 & 0 & 0 & 0 & H_{1}(s) & H_{2}(s) \\
0 & 0 & 0 & 0 & 0 & 0 & 0 & 0 & 0 & 0 \\
0 & 0 & 0 & 0 & H_{1, s}(s) & H_{2, s}(s) & 0 & 0 & 0 & 0 \\
0 & 0 & 0 & 0 & 0 & 0 & 0 & 0 & 0 & 0 \\
0 & 0 & 0 & 0 & 0 & 0 & H_{1, s}(s) & H_{2, s}(s) & 0 & 0 \\
0 & 0 & 0 & 0 & 0 & 0 & 0 & 0 & 0 & 0 \\
0 & 0 & 0 & 0 & 0 & 0 & 0 & 0 & H_{1, s}(s) & H_{2, s}(s) \\
0 & 0 & 0 & 0 & \frac{3 M_{3}(s)}{2 \ell_{k}} & -\frac{3 M_{3}(s)}{2 \ell_{k}} & 0 & 0 & \frac{6 M_{3}(s)}{5 \ell_{k}} & -\frac{6 M_{3}(s)}{5 \ell_{k}} \\
0 & 0 & 0 & 0 & 0 & 0 & 0 & 0 & 0 & 0
\end{array}\right.
$$

(continued on next page) 


$$
\begin{aligned}
& \begin{array}{cccccccccc}
0 & 0 & 0 & 0 & 0 & 0 & 0 & 0 & 0 & 0 \\
0 & 0 & 0 & 0 & 0 & 0 & 0 & 0 & 0 & 0 \\
0 & 0 & H_{3}(s) & H_{4}(s) & 0 & 0 & 0 & 0 & 0 & 0 \\
0 & 0 & 0 & 0 & 0 & 0 & H_{3}(s) & H_{4}(s) & 0 & 0 \\
0 & 0 & 0 & 0 & 0 & 0 & 0 & 0 & 0 & 0 \\
N_{1}(s) & N_{2}(s) & 0 & 0 & 0 & 0 & 0 & 0 & 0 & 0 \\
0 & 0 & H_{3, s}(s) & H_{4, s}(s) & 0 & 0 & 0 & 0 & 0 & 0 \\
0 & 0 & 0 & 0 & N_{1}(s) & N_{2}(s) & 0 & 0 & 0 & 0 \\
0 & 0 & 0 & 0 & 0 & 0 & H_{3, s}(s) & H_{4, s}(s) & 0 & 0 \\
0 & 0 & 0 & 0 & 0 & 0 & 0 & 0 & N_{1}(s) & N_{2}(s) \\
0 & 0 & 0 & 0 & 0 & 0 & 0 & 0 & 0 & 0 \\
0 & 0 & \frac{3 M_{3}(s)}{4} & \frac{3 M_{3}(s)}{4} & 0 & 0 & 0 & 0 & 0 & 0 \\
0 & 0 & 0 & 0 & 0 & 0 & 0 & 0 & 0 & 0
\end{array} \\
& \left.\begin{array}{cccccc}
0 & 0 & 0 & 0 & 0 & 0 \\
0 & 0 & 0 & 0 & 0 & 0 \\
0 & 0 & 0 & 0 & 0 & 0 \\
0 & 0 & 0 & 0 & 0 & 0 \\
H_{3}(s) & H_{4}(s) & 0 & 0 & 0 & 0 \\
0 & 0 & 0 & 0 & 0 & 0 \\
0 & 0 & 0 & 0 & 0 & 0 \\
0 & 0 & 0 & 0 & 0 & 0 \\
0 & 0 & 0 & 0 & 0 & 0 \\
0 & 0 & 0 & 0 & 0 & 0 \\
H_{3, s}(s) & H_{4, s}(s) & 0 & 0 & 0 & 0 \\
\frac{3 M_{3}(s)}{5} & \frac{3 M_{3}(s)}{5} & M_{1}(s)+\frac{M_{3}(s)}{2} & M_{1}(s)+\frac{M_{3}(s)}{2} & 0 & 0 \\
0 & 0 & 0 & 0 & N_{1}(s) & N_{2}(s)
\end{array}\right]
\end{aligned}
$$

The matrix $\mathbf{Z}^{(k)}$ in Eq. (39), which is the transformation matrix between the nodal degrees of freedom defined in the element $(x, y)$ and line coordinate $(n, s)$ systems for the $k^{\text {th }}$ edge, is defined as

$$
\begin{aligned}
& \mathbf{Z}^{(1)}=\left[\mathbf{Z}_{1}, \mathbf{Z}_{2}, 0\right] \\
& \mathbf{Z}^{(2)}=\left[0, \mathbf{Z}_{1}, \mathbf{Z}_{2}\right] \\
& \mathbf{Z}^{(3)}=\left[\mathbf{Z}_{2}, 0, \mathbf{Z}_{1}\right]
\end{aligned}
$$

where $\mathbf{Z}_{1}$ and $\mathbf{Z}_{2}$ are defined below in terms of $c_{k}$ and $s_{k}$ representing $c_{k}=\cos \left(\varphi_{k}\right)$ and $s_{k}=\sin \left(\varphi_{k}\right)$, respectively 


$$
\mathbf{Z}_{1}=\left[\begin{array}{ccccccccccccc}
c_{k} & s_{k} & 0 & 0 & 0 & 0 & 0 & 0 & 0 & 0 & 0 & 0 & 0 \\
0 & 0 & 0 & 0 & 0 & 0 & 0 & 0 & 0 & 0 & 0 & 0 & 0 \\
-s_{k} & c_{k} & 0 & 0 & 0 & 0 & 0 & 0 & 0 & 0 & 0 & 0 & 0 \\
0 & 0 & 0 & 0 & 0 & 0 & 0 & 0 & 0 & 0 & 0 & 0 & 0 \\
0 & 0 & 1 & 0 & 0 & 0 & 0 & 0 & 0 & 0 & 0 & 0 & 0 \\
0 & 0 & 0 & 0 & 0 & 0 & 0 & 0 & 0 & 0 & 0 & 0 & 0 \\
0 & 0 & 0 & 1 & 0 & 0 & 0 & 0 & 0 & 0 & 0 & 0 & 0 \\
0 & 0 & 0 & 0 & 0 & 0 & 0 & 0 & 0 & 0 & 0 & 0 & 0 \\
0 & 0 & 0 & 0 & 1 & 0 & 0 & 0 & 0 & 0 & 0 & 0 & 0 \\
0 & 0 & 0 & 0 & 0 & 0 & 0 & 0 & 0 & 0 & 0 & 0 & 0 \\
0 & 0 & 0 & 0 & 0 & c_{k} & s_{k} & 0 & 0 & 0 & 0 & 0 & 0 \\
0 & 0 & 0 & 0 & 0 & 0 & 0 & 0 & 0 & 0 & 0 & 0 & 0 \\
0 & 0 & 0 & 0 & 0 & 0 & 0 & c_{k} & s_{k} & 0 & 0 & 0 & 0 \\
0 & 0 & 0 & 0 & 0 & 0 & 0 & 0 & 0 & 0 & 0 & 0 & 0 \\
0 & 0 & 0 & 0 & 0 & 0 & 0 & 0 & 0 & c_{k} & s_{k} & 0 & 0 \\
0 & 0 & 0 & 0 & 0 & 0 & 0 & 0 & 0 & 0 & 0 & 0 & 0 \\
0 & 0 & 0 & 0 & 0 & -s_{k} & c_{k} & 0 & 0 & 0 & 0 & 0 & 0 \\
0 & 0 & 0 & 0 & 0 & 0 & 0 & 0 & 0 & 0 & 0 & 0 & 0 \\
0 & 0 & 0 & 0 & 0 & 0 & 0 & -s_{k} & c_{k} & 0 & 0 & 0 & 0 \\
0 & 0 & 0 & 0 & 0 & 0 & 0 & 0 & 0 & 0 & 0 & 0 & 0 \\
0 & 0 & 0 & 0 & 0 & 0 & 0 & 0 & 0 & -s_{k} & c_{k} & 0 & 0 \\
0 & 0 & 0 & 0 & 0 & 0 & 0 & 0 & 0 & 0 & 0 & 0 & 0 \\
0 & 0 & 0 & 0 & 0 & 0 & 0 & 0 & 0 & 0 & 0 & -c_{k} & -s_{k} \\
0 & 0 & 0 & 0 & 0 & 0 & 0 & 0 & 0 & 0 & 0 & 0 & 0 \\
0 & 0 & 0 & 0 & 0 & 0 & 0 & 0 & 0 & 0 & 0 & -s_{k} & c_{k} \\
0 & 0 & 0 & 0 & 0 & 0 & 0 & 0 & 0 & 0 & 0 & 0 & 0
\end{array}\right]
$$

and 


$$
\mathbf{Z}_{2}=\left[\begin{array}{ccccccccccccc}
0 & 0 & 0 & 0 & 0 & 0 & 0 & 0 & 0 & 0 & 0 & 0 & 0 \\
c_{k} & s_{k} & 0 & 0 & 0 & 0 & 0 & 0 & 0 & 0 & 0 & 0 & 0 \\
0 & 0 & 0 & 0 & 0 & 0 & 0 & 0 & 0 & 0 & 0 & 0 & 0 \\
-s_{k} & c_{k} & 0 & 0 & 0 & 0 & 0 & 0 & 0 & 0 & 0 & 0 & 0 \\
0 & 0 & 0 & 0 & 0 & 0 & 0 & 0 & 0 & 0 & 0 & 0 & 0 \\
0 & 0 & 1 & 0 & 0 & 0 & 0 & 0 & 0 & 0 & 0 & 0 & 0 \\
0 & 0 & 0 & 0 & 0 & 0 & 0 & 0 & 0 & 0 & 0 & 0 & 0 \\
0 & 0 & 0 & 1 & 0 & 0 & 0 & 0 & 0 & 0 & 0 & 0 & 0 \\
0 & 0 & 0 & 0 & 0 & 0 & 0 & 0 & 0 & 0 & 0 & 0 & 0 \\
0 & 0 & 0 & 0 & 1 & 0 & 0 & 0 & 0 & 0 & 0 & 0 & 0 \\
0 & 0 & 0 & 0 & 0 & 0 & 0 & 0 & 0 & 0 & 0 & 0 & 0 \\
0 & 0 & 0 & 0 & 0 & c_{k} & s_{k} & 0 & 0 & 0 & 0 & 0 & 0 \\
0 & 0 & 0 & 0 & 0 & 0 & 0 & 0 & 0 & 0 & 0 & 0 & 0 \\
0 & 0 & 0 & 0 & 0 & 0 & 0 & c_{k} & s_{k} & 0 & 0 & 0 & 0 \\
0 & 0 & 0 & 0 & 0 & 0 & 0 & 0 & 0 & 0 & 0 & 0 & 0 \\
0 & 0 & 0 & 0 & 0 & 0 & 0 & 0 & 0 & c_{k} & s_{k} & 0 & 0 \\
0 & 0 & 0 & 0 & 0 & 0 & 0 & 0 & 0 & 0 & 0 & 0 & 0 \\
0 & 0 & 0 & 0 & 0 & -s_{k} & c_{k} & 0 & 0 & 0 & 0 & 0 & 0 \\
0 & 0 & 0 & 0 & 0 & 0 & 0 & 0 & 0 & 0 & 0 & 0 & 0 \\
0 & 0 & 0 & 0 & 0 & 0 & 0 & -s_{k} & c_{k} & 0 & 0 & 0 & 0 \\
0 & 0 & 0 & 0 & 0 & 0 & 0 & 0 & 0 & 0 & 0 & 0 & 0 \\
0 & 0 & 0 & 0 & 0 & 0 & 0 & 0 & 0 & -s_{k} & c_{k} & 0 & 0 \\
0 & 0 & 0 & 0 & 0 & 0 & 0 & 0 & 0 & 0 & 0 & 0 & 0 \\
0 & 0 & 0 & 0 & 0 & 0 & 0 & 0 & 0 & 0 & 0 & -c_{k} & -s_{k} \\
0 & 0 & 0 & 0 & 0 & 0 & 0 & 0 & 0 & 0 & 0 & 0 & 0 \\
0 & 0 & 0 & 0 & 0 & 0 & 0 & 0 & 0 & 0 & 0 & -s_{k} & c_{k}
\end{array}\right]
$$

The transformation between the displacement vectors, $\mathbf{u}^{\prime(k)}$ and $\mathbf{u}^{(k)}$, is established as $\mathbf{u}^{\prime(k)}=\mathbf{Y}^{(k)} \mathbf{u}^{(k)}$, where the matrix $\mathbf{Y}^{(k)}$ is defined as

$$
\mathbf{Y}^{(k)}=\left[\begin{array}{ccccccccccccc}
c_{k} & s_{k} & 0 & 0 & 0 & 0 & 0 & 0 & 0 & 0 & 0 & 0 & 0 \\
-s_{k} & c_{k} & 0 & 0 & 0 & 0 & 0 & 0 & 0 & 0 & 0 & 0 & 0 \\
0 & 0 & 1 & 0 & 0 & 0 & 0 & 0 & 0 & 0 & 0 & 0 & 0 \\
0 & 0 & 0 & 1 & 0 & 0 & 0 & 0 & 0 & 0 & 0 & 0 & 0 \\
0 & 0 & 0 & 0 & 1 & 0 & 0 & 0 & 0 & 0 & 0 & 0 & 0 \\
0 & 0 & 0 & 0 & 0 & c_{k} & s_{k} & 0 & 0 & 0 & 0 & 0 & 0 \\
0 & 0 & 0 & 0 & 0 & -s_{k} & c_{k} & 0 & 0 & 0 & 0 & 0 & 0 \\
0 & 0 & 0 & 0 & 0 & 0 & 0 & c_{k} & s_{k} & 0 & 0 & 0 & 0 \\
0 & 0 & 0 & 0 & 0 & 0 & 0 & -s_{k} & c_{k} & 0 & 0 & 0 & 0 \\
0 & 0 & 0 & 0 & 0 & 0 & 0 & 0 & 0 & c_{k} & s_{k} & 0 & 0 \\
0 & 0 & 0 & 0 & 0 & 0 & 0 & 0 & 0 & -s_{k} & c_{k} & 0 & 0 \\
0 & 0 & 0 & 0 & 0 & 0 & 0 & 0 & 0 & 0 & 0 & c_{k} & s_{k} \\
0 & 0 & 0 & 0 & 0 & 0 & 0 & 0 & 0 & 0 & 0 & -s_{k} & c_{k}
\end{array}\right]
$$


The matrix $\mathbf{B}_{\mathbf{u}}$ in Eq. (47) is defined as

$$
\mathbf{B}_{\mathbf{u}}=\left[\begin{array}{ccccccccccccc}
1 & 0 & 0 & 0 & 0 & 0 & 0 & 0 & 0 & 0 & 0 & 0 & 0 \\
0 & 1 & 0 & 0 & 0 & 0 & 0 & 0 & 0 & 0 & 0 & 0 & 0 \\
0 & 0 & 1 & 0 & 0 & 0 & 0 & 0 & 0 & 0 & 0 & 0 & 0 \\
0 & 0 & 0 & 0 & 0 & 0 & 0 & 0 & 0 & 0 & 0 & 1 & 0 \\
0 & 0 & 0 & 0 & 0 & 0 & 0 & 0 & 0 & 0 & 0 & 0 & 1 \\
0 & 0 & 0 & 1 & 0 & 0 & 0 & 0 & 0 & 0 & 0 & 0 & 0 \\
0 & 0 & 0 & 0 & 0 & 0 & 0 & h & 0 & 0 & 0 & 0 & 0 \\
0 & 0 & 0 & 0 & 0 & 0 & 0 & 0 & h & 0 & 0 & 0 & 0 \\
0 & 0 & 0 & 0 & 1 & 0 & 0 & 0 & 0 & 0 & 0 & 0 & 0 \\
0 & 0 & 0 & 0 & 0 & 5 / 4 & 0 & 0 & 0 & 1 & 0 & 0 & 5 / 4 \\
0 & 0 & 0 & 0 & 0 & 0 & 5 / 4 & 0 & 0 & 0 & 1 & 5 / 4 & 0
\end{array}\right]
$$

The matrix $\mathbf{B}_{\mathrm{s}}$ in Eq. (48), which relates the boundary stress vector $\mathbf{T}_{b}^{(k)}$ to the resultant stress vector defined in the element, is defined as

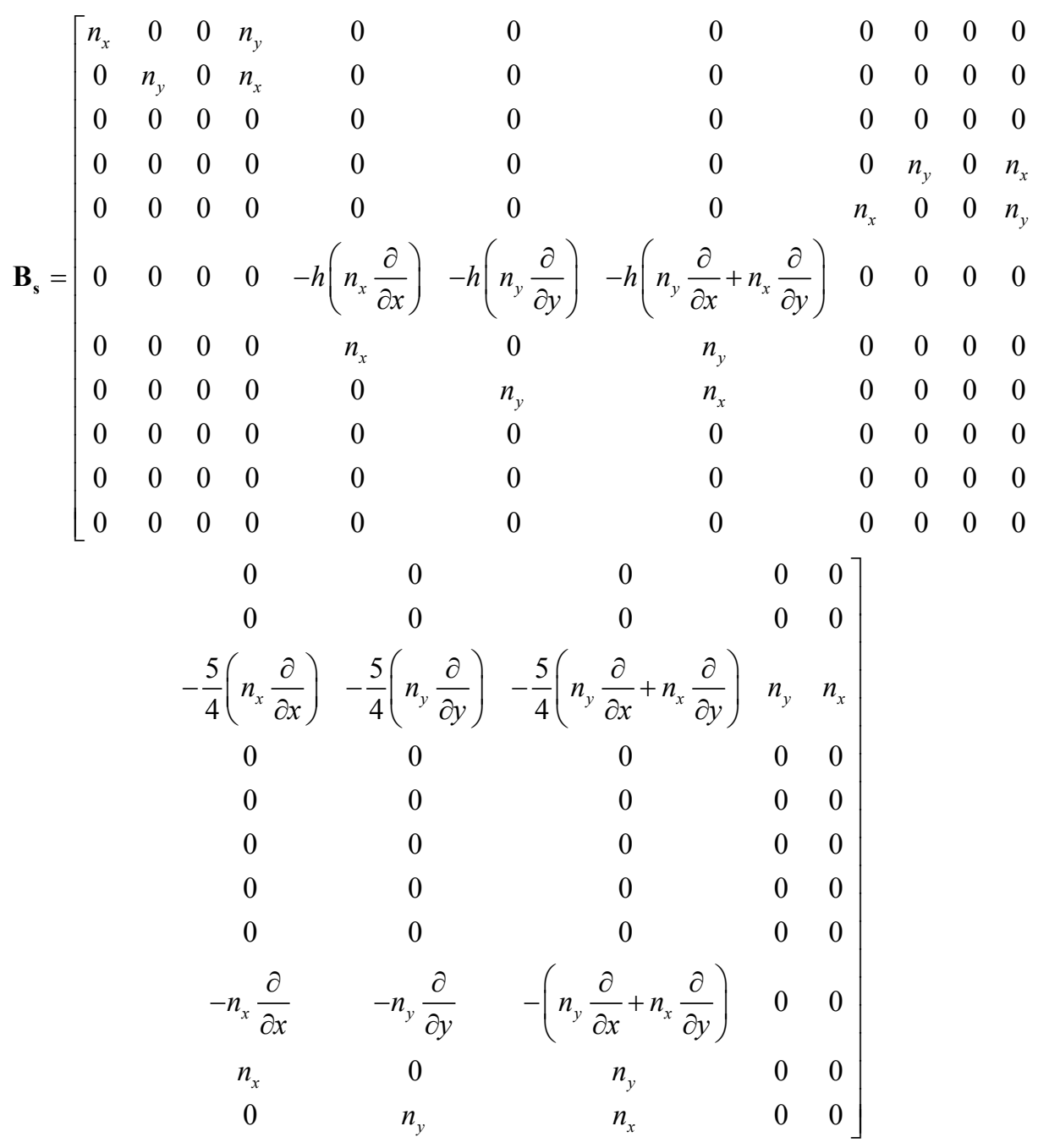


The components of the resultant stress vector, $\mathbf{S}_{h}$, given in Eq. (29) satisfy the equilibrium equations provided that the following constraint conditions are imposed in order to eliminate the redundancy of the assumed stress field:

$$
\begin{gathered}
\operatorname{Re}\left[\sum_{k=1}^{4} z^{k-1}\left(k^{(0)} \alpha_{x x(k)}+i k^{(0)} \alpha_{x y(k)}\right)\right]=0 \\
\operatorname{Re}\left[\sum_{k=1}^{4} z^{k-1}\left(i k^{(0)} \alpha_{y y(k)}+k^{(0)} \alpha_{x y(k)}\right)\right]=0 \\
\operatorname{Re}\left[\frac{5}{4}\left(\sum_{k=2}^{4} z^{k-2}\left(k(k-1)^{(1)} \beta_{x x(k)}-k(k-1)^{(1)} \beta_{y y(k)}+2 i k(k-1)^{(1)} \beta_{x y(k)}\right)\right)\right. \\
\left.-\left(\sum_{k=1}^{3} z^{k-1}\left(k^{(0)} \rho_{x z(k)}+i k^{(0)} \rho_{y z(k)}\right)\right)\right]=0 \\
\operatorname{Re}\left[\frac{5}{4}\left(\sum_{k=1}^{4} z^{k-1}\left(i k^{(1)} \beta_{y y(k)}+k^{(1)} \beta_{x y(k)}\right)\right)+\left(\sum_{k=1}^{4} z^{k-1}\left(i k^{(0)} \beta_{y y(k)}+k^{(0)} \beta_{x y(k)}\right)\right)\right. \\
\left.\operatorname{Re}\left[\frac{5}{4}\left(\sum_{k=1}^{3} z^{k} z^{(0)} \rho_{y z(k)}\right)\right)\right]=0 \\
\left.-\left(\sum_{k=0}^{3} z^{k}\left({ }^{(0)} \rho_{x z(k)}\right)\right)\right]=0 \\
\operatorname{Re}\left[\left(\sum_{k=2}^{4} z^{k-2}\left(k(k-1)^{(1)} \beta_{x x(k)}-k(k-1)^{(1)} \beta_{y y(k)}+2 i k(k-1)^{(1)} \beta_{x y(k)}\right)\right)\right. \\
\operatorname{Re}\left[h\left(\sum_{k=2}^{4} z^{k-2}\left(k(k-1)^{(1)} \alpha_{x x(k)}-k(k-1)^{(1)} \alpha_{y y(k)}+2 i k(k-1)^{(1)} \alpha_{x y(k)}\right)\right)\right. \\
\left.h^{2}\left(\sum_{k=0}^{2} z^{k(0)} \beta_{z z(k)} z^{k(0)} \alpha_{z z(k)}\right)\right]=0 \\
\left.\sum_{k=1}^{4} z^{k-1}\left(k^{(0)} \beta_{x x(k)}+i k^{(0)} \beta_{x y(k)}\right)\right)
\end{gathered}
$$

The relations used in the construction of matrix $\mathbf{c}$ in Eq. (30) and also defined explicitly in terms of complex variables in Eq. (C8) are imposed as constraint equations that are suitable for computational implementation as follows:

$$
\begin{array}{lr}
k \operatorname{Re}\left[{ }^{(0)} \alpha_{x x(k)}\right]-k \operatorname{Im}\left[{ }^{(0)} \alpha_{x y(k)}\right]=0 & (k=1,2,3,4)(\mathrm{C} 9 \mathrm{a}) \\
k \operatorname{Im}\left[{ }^{(0)} \alpha_{x x(k)}\right]+k \operatorname{Re}\left[{ }^{(0)} \alpha_{x y(k)}\right]=0 & (k=2,3,4)(\mathrm{C} 9 \mathrm{~b}) \\
-k \operatorname{Im}\left[{ }^{(0)} \alpha_{y y(k)}\right]+\operatorname{Re}\left[{ }^{(0)} \alpha_{x y(k)}\right]=0 & (k=1,2,3,4)(\mathrm{C} 9 \mathrm{c}) \\
k \operatorname{Re}\left[{ }^{(0)} \alpha_{y y(k)}\right]+\operatorname{Im}\left[{ }^{(0)} \alpha_{x y(k)}\right]=0 & (k=2,3,4)(\mathrm{C} 9 \mathrm{~d})
\end{array}
$$




$$
\begin{aligned}
& \frac{5}{4}\left(k(k-1) \operatorname{Re}\left[{ }^{(1)} \beta_{x x(k)}\right]-k(k-1) \operatorname{Re}\left[{ }^{(1)} \beta_{y y(k)}\right]-2 k(k-1) \operatorname{Im}\left[{ }^{(1)} \beta_{x y(k)}\right]\right) \\
& -\left((k-1) \operatorname{Re}\left[{ }^{(0)} \rho_{x z(k-1)}\right]-(k-1) \operatorname{Im}\left[{ }^{(0)} \rho_{y z(k-1)}\right]\right)=0 \\
& \frac{5}{4}\left(k(k-1) \operatorname{Im}\left[{ }^{(1)} \beta_{x x(k)}\right]-k(k-1) \operatorname{Im}\left[{ }^{(1)} \beta_{y y(k)}\right]+2 k(k-1) \operatorname{Re}\left[{ }^{(1)} \beta_{x y(k)}\right]\right) \\
& -\left((k-1) \operatorname{Im}\left[{ }^{(0)} \rho_{x z(k-1)}\right]+(k-1) \operatorname{Re}\left[{ }^{(0)} \rho_{y z(k-1)}\right]\right)=0 \\
& \frac{5}{4}\left(-k \operatorname{Im}\left[{ }^{(1)} \beta_{y y(k)}\right]+k \operatorname{Re}\left[{ }^{(1)} \beta_{x y(k)}\right]\right)+\left(-k \operatorname{Im}\left[{ }^{(0)} \beta_{y y(k)}\right]+k \operatorname{Re}\left[{ }^{(0)} \beta_{x y(k)}\right]\right) \quad(k=1,2,3,4) \quad(\mathrm{C} 9 \mathrm{~g}) \\
& -\operatorname{Re}\left[{ }^{(0)} \rho_{y z(k-1)}\right]=0 \\
& \frac{5}{4}\left(k \operatorname{Re}\left[{ }^{(1)} \beta_{y y(k)}\right]+k \operatorname{Im}\left[{ }^{(1)} \beta_{x y(k)}\right]\right)+\left(k \operatorname{Re}\left[{ }^{(0)} \beta_{y y(k)}\right]+k \operatorname{Im}\left[{ }^{(0)} \beta_{x y(k)}\right]\right) \\
& -\operatorname{Im}\left[{ }^{(0)} \rho_{y z(k-1)}\right]=0 \\
& \frac{5}{4}\left(k \operatorname{Re}\left[{ }^{(1)} \beta_{x x(k)}\right]-k \operatorname{Im}\left[{ }^{(1)} \beta_{x y(k)}\right]\right)+\left(k \operatorname{Re}\left[{ }^{(0)} \beta_{x x(k)}\right]-k \operatorname{Im}\left[{ }^{(0)} \beta_{x y(k)}\right]\right) \\
& -\operatorname{Re}\left[{ }^{(0)} \rho_{x z(k-1)}\right]=0 \\
& \frac{5}{4}\left(k \operatorname{Im}\left[{ }^{(1)} \beta_{x x(k)}\right]+k \operatorname{Re}\left[{ }^{(1)} \beta_{x y(k)}\right]\right)+\left(k \operatorname{Im}\left[{ }^{(0)} \beta_{x x(k)}\right]+k \operatorname{Re}\left[{ }^{(0)} \beta_{x y(k)}\right]\right) \\
& -\operatorname{Im}\left[{ }^{(0)} \rho_{x z(k-1)}\right]=0 \\
& \frac{1}{h}\left(\operatorname{Re}\left[{ }^{(0)} \alpha_{z z(k-2)}\right]\right)+h\left(\left(k(k-1) \operatorname{Re}\left[{ }^{(1)} \alpha_{x x(k)}\right]-k(k-1) \operatorname{Re}\left[{ }^{(1)} \alpha_{y y(k)}\right]\right.\right. \\
& \left.-2 k(k-1) \operatorname{Im}\left[{ }^{(1)} \alpha_{x y(k)}\right]\right)=0 \\
& \frac{1}{h}\left(\operatorname{Im}\left[{ }^{(0)} \alpha_{z z(k-2)}\right]\right)+h\left(\left(k(k-1) \operatorname{Im}\left[{ }^{(1)} \alpha_{x x(k)}\right]-k(k-1) \operatorname{Im}\left[{ }^{(1)} \alpha_{y y(k)}\right]\right.\right. \\
& \left.+2 k(k-1) \operatorname{Re}\left[{ }^{(1)} \alpha_{x y(k)}\right]\right)=0 \\
& \frac{1}{h^{2}}\left(\operatorname{Re}\left[{ }^{(0)} \beta_{z z(k-2)}\right]\right)+\left(\left(k(k-1) \operatorname{Re}\left[{ }^{(1)} \beta_{x x(k)}\right]-k(k-1) \operatorname{Re}\left[{ }^{(1)} \beta_{y y(k)}\right] \quad(k=2,3,4)(\mathrm{C} 9 \mathrm{~m})\right.\right. \\
& \left.-2 k(k-1) \operatorname{Im}\left[{ }^{(1)} \beta_{x y(k)}\right]\right)=0 \\
& \frac{1}{h^{2}}\left(\operatorname{Im}\left[{ }^{(0)} \beta_{z z(k-2)}\right]\right)+\left(\left(k(k-1) \operatorname{Im}\left[{ }^{(1)} \beta_{x x(k)}\right]-k(k-1) \operatorname{Im}\left[{ }^{(1)} \beta_{y y(k)}\right]\right.\right. \\
& \left.+2 k(k-1) \operatorname{Re}\left[{ }^{(1)} \beta_{x y(k)}\right]\right)=0
\end{aligned}
$$

\section{Appendix D}

The transverse shear stresses, $\sigma_{x z}^{(k)}$ and $\sigma_{y z}^{(k)}$, in the $k^{\text {th }}$ layer are obtained by integrating the in-plane equilibrium equations while utilizing the constitutive relations given in Eq. (6) as

$$
\sigma_{x z}^{(k)}=-\int_{-h / 2}^{z^{(k)}}\left(\sigma_{x x, x}^{(k)}+\sigma_{x y, y}^{(k)}\right) d \xi
$$




$$
\sigma_{y z}^{(k)}=-\int_{-h / 2}^{z^{(k)}}\left(\sigma_{x y, x}^{(k)}+\sigma_{y y, y}^{(k)}\right) d \xi
$$

in which the derivatives of in-plane stresses are expressed as the dot products

$$
\begin{aligned}
& \sigma_{x x, x}^{(k)}=\left(\bar{C}_{11}^{(k)}, \quad \bar{C}_{12}^{(k)}, \quad R_{13}^{(k)}, \quad \bar{C}_{16}^{(k)}\right)\left\lfloor\left(\varepsilon_{x x, x}-\alpha_{x x} \Delta T_{, x}, \quad \varepsilon_{y y, x}-\alpha_{y y} \Delta T_{, x}, \quad \sigma_{z z, x}, \quad \gamma_{x y, x}-\alpha_{x y} \Delta T_{, x}\right)\right. \\
& \sigma_{y y, y}^{(k)}=\left(\bar{C}_{12}^{(k)}, \quad \bar{C}_{22}^{(k)}, \quad R_{23}^{(k)}, \quad \bar{C}_{26}^{(k)}\right)\left\lfloor\left(\varepsilon_{x x, y}-\alpha_{x x} \Delta T_{, y}, \quad \varepsilon_{y y, y}-\alpha_{y y} \Delta T_{, y}, \quad \sigma_{z z, y}, \quad \gamma_{x y, y}-\alpha_{x y} \Delta T_{, y}\right)\right. \\
& \sigma_{x y, x}^{(k)}=\left(\begin{array}{llll}
(k) & \bar{C}_{16}^{(k)}, & R_{63}^{(k)}, & \bar{C}_{66}^{(k)}
\end{array}\right)\left(\varepsilon_{x x, x}-\alpha_{x x} \Delta T_{, x}, \quad \varepsilon_{y y, x}-\alpha_{y y} \Delta T_{, x}, \quad \sigma_{z z, x}, \quad \gamma_{x y, x}-\alpha_{x y} \Delta T_{, x}\right) \\
& \sigma_{x y, y}^{(k)}=\left(\bar{C}_{16}^{(k)}, \quad \bar{C}_{26}^{(k)}, \quad R_{63}^{(k)}, \quad \bar{C}_{66}^{(k)}\right)\left(\varepsilon_{x x, y}-\alpha_{x x} \Delta T_{, y}, \quad \varepsilon_{y y, y}-\alpha_{y y} \Delta T_{, y}, \quad \sigma_{z z, y}, \quad \gamma_{x y, y}-\alpha_{x y} \Delta T_{, y}\right)
\end{aligned}
$$

Note that the material properties $\bar{C}_{i j}^{(k)}$ and $R_{i j}^{(k)}$ are functions of $\xi^{(k)}$ (thickness direction) only. Similarly, the transverse stress component, $\sigma_{z z}^{(k)}$, is obtained by integrating the transverse equilibrium equation as

$$
\sigma_{z z}^{(k)}=-\int_{-h / 2}^{z^{(k)}}\left(\sigma_{x z, x}^{(k)}+\sigma_{y z, y}^{(k)}\right) d \xi=\int_{-h / 2}^{z^{(k)}} \int_{-h / 2}^{z^{(k)}}\left(\sigma_{x x, x x}^{(k)}+2 \sigma_{x y, x y}^{(k)}+\sigma_{y y, y y}^{(k)}\right) d \eta d \xi
$$

in which the double derivatives of $\sigma_{x x}^{(k)}, \sigma_{y y}^{(k)}$, and $\sigma_{x y}^{(k)}$ are expressed as the dot products

$$
\begin{aligned}
& \sigma_{x x, x x}^{(k)}=\left(\bar{C}_{11}^{(k)}, \quad \bar{C}_{12}^{(k)}, \quad R_{13}^{(k)}, \quad \bar{C}_{16}^{(k)}\right)\left(\varepsilon_{x x, x x}-\alpha_{x x} \Delta T_{, x x}, \quad \varepsilon_{y y, x x}-\alpha_{y y} \Delta T_{, x x}, \quad \sigma_{z z, x x}, \quad \gamma_{x y, x x}-\alpha_{x y} \Delta T_{, x x}\right) \\
& \sigma_{y y, y y}^{(k)}=\left(\bar{C}_{12}^{(k)}, \quad \bar{C}_{22}^{(k)}, \quad R_{23}^{(k)}, \quad \bar{C}_{26}^{(k)}\right)\left(\varepsilon_{x x, y y}-\alpha_{x x} \Delta T_{, y y}, \quad \varepsilon_{y y, y y}-\alpha_{y y} \Delta T_{, y y}, \quad \sigma_{z z, y y}, \quad \gamma_{x y, y y}-\alpha_{x y} \Delta T_{, y y}\right) \\
& \sigma_{x y, x y}^{(k)}=\left(\bar{C}_{16}^{(k)}, \quad \bar{C}_{26}^{(k)}, \quad R_{63}^{(k)}, \quad \bar{C}_{66}^{(k)}\right) \llbracket\left(\varepsilon_{x x, x y}-\alpha_{x x} \Delta T_{, x y}, \quad \varepsilon_{y y, x y}-\alpha_{y y} \Delta T_{, x y}, \quad \sigma_{z z, x y}, \quad \gamma_{x y, x y}-\alpha_{x y} \Delta T_{, x y}\right)
\end{aligned}
$$

The integration of the in-plane and transverse equilibrium equations requires the continuous representation of the strain components and the transverse normal stress component so that their differentiation can be performed. The nodal degrees of freedom obtained from the solution of Eq. (61) are then utilized to approximate their continuous behavior in each element by employing linear interpolation functions, $N_{m}(x, y)$, as

$$
\begin{aligned}
& {\left[u^{(e)}, v^{(e)}, w^{(e)}, w_{1}^{(e)}, w_{2}^{(e)}, w_{, x}^{(e)}, w_{, y}^{(e)}, w_{1, x}^{(e)}, w_{1, y}^{(e)}, w_{2, x}^{(e)}, w_{2, y}^{(e)}, \theta_{x}^{(e)}, \theta_{y}^{(e)}\right]=\sum_{m=1}^{3} N_{m}(x, y)} \\
& \quad\left[u_{(m)}^{(e)}, v_{(m)}^{(e)}, w_{(m)}^{(e)}, w_{1(m)}^{(e)}, w_{2(m)}^{(e)}, w_{, x(m)}^{(e)}, w_{, y(m)}^{(e)}, w_{1, x(m)}^{(e)}, w_{1, y(m)}^{(e)}, w_{2, x(m)}^{(e)} w_{2, y(m)}^{(e)}, \theta_{x(m)}^{(e)}, \theta_{y(m)}^{(e)}\right]
\end{aligned}
$$

in which the quantities with subscript $(m)$ denote their value at node " $m$."

However, for simply supported panels, their global behavior can be approximated in terms of Fourier series as

$$
\begin{gathered}
\tilde{u}(x, y)=\sum_{m=1}^{\infty} \sum_{n=1}^{\infty} U^{m n} \cos \left(\alpha_{m} x\right) \sin \left(\beta_{n} y\right) \\
\tilde{v}(x, y)=\sum_{m=1}^{\infty} \sum_{n=1}^{\infty} V^{m n} \sin \left(\alpha_{m} x\right) \cos \left(\beta_{n} y\right) \\
{\left[\tilde{w}(x, y), \tilde{w}_{1}(x, y), \tilde{w}_{2}(x, y)\right]=\sum_{m=1}^{\infty} \sum_{n=1}^{\infty}\left[W^{m n}, W_{1}^{m n}, W_{2}^{m n}\right] \sin \left(\alpha_{m} x\right) \sin \left(\beta_{n} y\right)} \\
{\left[\tilde{w}_{, x}(x, y), \tilde{w}_{1, x}(x, y), \tilde{w}_{2, x}(x, y)\right]=\sum_{m=1}^{\infty} \sum_{n=1}^{\infty}\left[W_{, x}^{m n}, W_{1, x}^{m n}, W_{2, x}^{m n}\right] \cos \left(\alpha_{m} x\right) \sin \left(\beta_{n} y\right)}
\end{gathered}
$$




$$
\begin{gathered}
{\left[\tilde{w}_{, y}(x, y), \tilde{w}_{1, y}(x, y), \tilde{w}_{2, y}(x, y)\right]=\sum_{m=1}^{\infty} \sum_{n=1}^{\infty}\left[W_{, y}^{m n}, W_{1, y}^{m n}, W_{2, y}^{m n}\right] \sin \left(\alpha_{m} x\right) \cos \left(\beta_{n} y\right)} \\
\tilde{\theta}_{x}(x, y)=\sum_{m=1}^{\infty} \sum_{n=1}^{\infty} \Phi_{x}^{m n} \sin \left(\alpha_{m} x\right) \cos \left(\beta_{n} y\right) \\
\tilde{\theta}_{y}(x, y)=\sum_{m=1}^{\infty} \sum_{n=1}^{\infty} \Phi_{y}^{m n} \cos \left(\alpha_{m} x\right) \sin \left(\beta_{n} y\right)
\end{gathered}
$$

where $\alpha_{m}$ and $\beta_{n}$ are defined as $m \pi /(2 a)$ and $n \pi /(2 b)$, respectively. The unknown coefficients are calculated by employing Gauss integration techniques

$$
\begin{aligned}
& U^{m n}=\frac{1}{a b} \sum_{e=1}^{N_{e}} \sum_{i=1}^{G} A^{(e)} u^{(e)}\left(x_{i}, y_{i}\right) \cos \left(\alpha_{m} x_{i}\right) \sin \left(\beta_{n} y_{i}\right) \psi_{i} \\
& V^{m n}=\frac{1}{a b} \sum_{e=1}^{N_{e}} \sum_{i=1}^{G} A^{(e)} v^{(e)}\left(x_{i}, y_{i}\right) \sin \left(\alpha_{m} x_{i}\right) \cos \left(\beta_{n} y_{i}\right) \psi_{i} \\
& {\left[W^{m n}, W_{1}^{m n}, W_{2}^{m n}\right]=\frac{1}{a b} \sum_{e=1}^{N_{e}} \sum_{i=1}^{G}\left[w^{(e)}(x, y), w_{1}^{(e)}(x, y), w_{2}^{(e)}(x, y)\right]} \\
& \sin \left(\alpha_{m} x_{i}\right) \sin \left(\beta_{n} y_{i}\right) \psi_{i} \\
& {\left[W_{, x}^{m n}, W_{1, x}^{m n}, W_{2, x}^{m n}\right]=\frac{1}{a b} \sum_{e=1}^{N_{e}} \sum_{i=1}^{G}\left[w_{, x}^{(e)}(x, y), w_{1, x}^{(e)}(x, y), w_{2, x}^{(e)}(x, y)\right]} \\
& \cos \left(\alpha_{m} x_{i}\right) \sin \left(\beta_{n} y_{i}\right) \psi_{i} \\
& {\left[W_{, y}^{m n}, W_{1, y}^{m n}, W_{2, y}^{m n}\right]=\frac{1}{a b} \sum_{e=1}^{N_{e}} \sum_{i=1}^{G}\left[w_{, y}^{(e)}(x, y), w_{1, y}^{(e)}(x, y), w_{2, y}^{(e)}(x, y)\right]} \\
& \sin \left(\alpha_{m} x_{i}\right) \cos \left(\beta_{n} y_{i}\right) \psi_{i} \\
& \Phi_{x}^{m n}=\frac{1}{a b} \sum_{e=1}^{N_{e}} \sum_{i=1}^{G} A^{(e)} \theta_{x}^{(e)}\left(x_{i}, y_{i}\right) \sin \left(\alpha_{m} x_{i}\right) \cos \left(\beta_{n} y_{i}\right) \psi_{i} \\
& \Phi_{y}^{m n}=\frac{1}{a b} \sum_{e=1}^{N_{e}} \sum_{i=1}^{G} A^{(e)} \theta_{y}^{(e)}\left(x_{i}, y_{i}\right) \cos \left(\alpha_{m} x_{i}\right) \sin \left(\beta_{n} y_{i}\right) \psi_{i}
\end{aligned}
$$

where $N_{e}$ is the number of elements, $G$ is the number of Gauss points for each element, and $\psi_{i}$ is the weight of the numerical integration at the $i^{\text {th }}$ Gauss point. The area of $e^{\text {th }}$ element, $A^{(e)}$, is given as

$$
A^{(e)}=\frac{1}{2}\left|\begin{array}{ccc}
1 & 1 & 1 \\
x_{1}^{(e)} & x_{2}^{(e)} & x_{3}^{(e)} \\
y_{1}^{(e)} & y_{2}^{(e)} & y_{3}^{(e)}
\end{array}\right|
$$

The Fourier coefficients are then used to obtain a continuous representation of the displacement components, using Eq. (D4), which can now be differentiated analytically. The second partial derivatives of the displacement components are used to calculate the first partial derivatives of the resultant strain and curvature components employing Eq. (5), which in turn are utilized to compute the transverse shear stress. Note that the computation of the transverse normal stress in Eq. (2a) requires the second derivative of the resultant strain and curvature components, therefore the third order derivatives of the displacement components in Eq. (D4) are evaluated.

Through-the-thickness variations of the displacement components are computed from the strain displacement relation as 


$$
\begin{gathered}
u_{\alpha}^{(k)}=\int_{-h / 2}^{z^{(k)}}\left(\gamma_{\alpha z}^{(k)}-u_{z, \alpha}\right) d \xi+u_{\alpha}(x, y,-h / 2) \\
=\int_{-h / 2}^{z^{(k)}}\left(\gamma_{\alpha z}^{(k)}-w_{, \alpha}(x, y)-w_{1, \alpha} \xi-w_{2, \alpha}\left(\xi^{2}-1 / 5\right)\right) d \xi+u_{\alpha}(x, y,-h / 2) \\
u_{z}^{(k)}=\int_{-h / 2}^{z^{(k)}} \varepsilon_{z z}^{(k)} d \xi+u_{z}(x, y,-h / 2)
\end{gathered}
$$

where $u_{\alpha}(x, y,-h / 2)$ and $u_{z}(x, y,-h / 2)$ are computed from the original displacement expression given in Eqs. (1) and (3). The strain components are obtained by the stress-strain relations.

\# 